\title{
Printed Works
}

Abe, Rudolf Horst: Die Erfurter medizinische Fakultät in den Jahren 1392-1524. Leipzig 1974.

Absmeier, Christine: Das schlesische Schulwesen im Jahrhundert der Reformation. Ständische Bildungsreformen im Geiste Philipp Melanchthons. Stuttgart 2011.

Achillini, Alessandro: Opera omnia in unum collecta cum annotationibus excellentissimi doctoris Pamphilii Montii Bononiensis. Venice 1545.

Adam, Melchior: Vitae Germanorum medicorum, qui seculo superiori, et quod excurrit, claruerunt. Heidelberg 1620.

Aëtius of Amida: Libri XVI. Vol. 3. Transl. by Giovanni Battista da Monte. Basel 1535.

Aewerdieck, Björn: Register zu den Wunderzeichenbüchern Job Fincels. Frankfurt am Main 2010.

Agasse, Jean-Michel: Introduction. In: idem and Concetta Pennuto (eds): Une correspondance entre deux médecins humanistes. Girolamo Mercuriale - Johann Crato von Krafftheim. Geneva 2016, pp. 9-133.

Agricola, Georgius: De ortu et causis subterraneorum libri V. De natura eorum quae effluunt ex terra libri IV. De natura fossilium libri X. De veteribus et novis metallis libri II. Bermannus sive de re metallica dialogus. Basel 1546.

Agrimi, Jole and Chiara Crisciani: Consilia médicaux. Turnhout 1994.

Aho, James: Confession and bookkeeping. The religious, moral, and rhetorical roots of modern accounting. Albany, NY 2005.

Albrecht, Stefan: Prag. In: Wolfgang Adam and Siegrid Westphal (eds): Handbuch kultureller Zentren der Frühen Neuzeit. Städte und Residenzen im alten deutschen Sprachraum. Berlin/Boston 2012, pp. 1649-1694.

Alciati, Andrea: Emblematum liber. Augsburg 1531.

Algazi, Gadi: Eine gelernte Lebensweise: Figurationen des Gelehrtenlebens zwischen Mittelalter und Früher Neuzeit. In: Berichte zur Wissenschaftsgeschichte 30 (2007), pp. 107-118.

Algazi, Gadi: Food for thought. Hieronymus Wolf grapples with the scholarly habitus. In: Rudolf Dekker (ed.): Egodocuments and history. Autobiographical writing in its social context since the Middle Ages. Hilversum 2002, pp. 21-44.

Algazi, Gadi: "Geistesabwesenheit”. Gelehrte zuhause um 1500. In: Alf Lüdtke and Reiner Prass (eds): Gelehrtenleben. Wissenschaftspraxis in der Neuzeit. Köln/Weimar/Vienna 2007, pp. 215-234.

Algazi, Gadi: Habitus, familia und forma vitae. Die Lebensweise mittelalterlicher Gelehrter in muslimischen, jüdischen und christlichen Gemeinden - vergleichend betrachtet. In: Frank Rexroth (ed.): Beiträge zur Kulturgeschichte der Gelehrten im späten Mittelalter. Ostfildern 2010, pp. 185-217.

Algazi, Gadi: Scholars in households. Refiguring the learned habitus, 1480-1550. In: Science in context 16 (2003), pp. 9-42.

Algazi, Gadi, Valentin Groebner and Bernhard Jussen (eds): Negotiating the gift. Pre-modern figurations of exchange. Göttingen 2003.

Alkemeyer, Thomas, Gunilla Budde and Dagmar Freist (eds): Selbst-Bildungen. Soziale und kulturelle Praktiken der Subjektivierung. Bielefeld 2013.

Alkemeyer, Thomas: Subjektivierung in sozialen Praktiken. Umrisse einer praxeologischen Analytik. In: Alkemeyer, Budde and Freist, Selbst-Bildungen (2013), pp. 33-68. 
Allen, Percy Stafford: Some letters of masters and scholars 1500-1530. In: The English historical review 22 (1907), pp. 740-754.

Alpinus, Prosper: De praesagienda vita et morte aegrotantium libri septem. Frankfurt 1601.

Amatus Lusitanus: Curationum medicinalium centuria prima, multiplici variaque rerum cognitione referta. Florence 1551.

Amatus Lusitanus: Curationum medicinalium centuria prima, multiplici variaque rerum cognitione referta. Paris 1552.

Amatus Lusitanus: Introitus ad aegrotantem. Simulque disgressio de crisi et diebus decretoribus. In: idem: Curationum (1552), pp. 1-61.

Alvarez, Antonio: Epistolarum et consiliorum medicinalium pars prima. Naples 1585.

Andreozzi, Alfonso: Le leggi penali degli antichi Chinesi: discorso proemiale sul diritto e sui limiti del punire e traduzioni originali dal cinese. Florence 1878.

Andreska, Jan: Losos labský v historických záznamech a v současnosti I. In: Živa (2010), pp. 178-182.

Andretta, Elisa and Marilyn Nicoud (eds): Être médecin à la cour (Italie, France, Espagne, XIIIe -XVIIIe siècle). Florence 2013.

Andretta, Elisa: Roma medica. Anatomie d'un système médical au XVIe siècle. Rome 2011.

Arbenz, Emil and Hermann Wartmann (eds): Die Vadianische Briefsammlung der

Stadtbibliothek St. Gallen. Part 6 and 7. St. Gallen 1906-1913.

Arber, Agnes: Herbals. Their origin and evolution. Cambridge 1986.

Arcaeus, Franciscus: De recta curandorum vulnerum ratione libri II. Antwerp 1574.

Argenterio, Giovanni: De morbis libri XIIII. Florence 1556.

Arrizabalaga, Jon, John Henderson and Roger French: The great pox. The French disease in Renaissance Europe. New Haven 1997.

Assion, Peter and Joachim Telle: Der Nürnberger Stadtarzt Johannes Magenbuch. Zu Leben und Werk eines Mediziners der Reformationszeit. In: Sudhoffs Archiv 56 (1972), pp. 353-421.

Augenio, Orazio: Epistolarum et consultationum medicinalium prioris tomi libri XII. Venice 1602.

Aumüller, Gerhard: Professor in Marburg und Leibarzt in Kassel? Lebensbilder hessischer Ärzte zur Zeit des Landgrafen Philipp (1504-1567) und die weitere Entwicklung der Medizin unter Landgraf Moritz (1572-1632). In: Irmtraut Sahmland and Kornelia Grundmann (eds): Perspektiven der Medizingeschichte Marburgs. Neue Studien und Kontexte. Darmstadt/ Marburg 2011, pp. 11-46.

Austrius, Sebastianus: De infantium sive puerorum morborum et symptomatorum dignitione tum curatione liber. Basel 1540.

Avicenna, Canon medicinae. Ed. and comm. by Giovanni Costeo. Venice 1595.

Baader, Gerhard: Medizinische Theorie und Praxis zwischen Arabismus und Renaissancehumanismus. In: Gundolf Keil, Bernd Moeller Bernd and Winfried Trusen (eds): Der Humanismus und die oberen Fakultäten. Weinheim 1987, pp. 185-213.

Bacchelli, Franco: Antonio Musa Brasavola archiatra di Ercole II duca di Ferrara. In: Micrologus 16 (2008), pp. 327-346.

Bachmann, Hans: Dr. Johann Peter Merenda. Aus dem Leben eines Innsbrucker Hofarztes, 1542 bis 1567. In: Tiroler Heimatblätter 28 (1953), pp. 5-10.

Bacon, Francis: Twoo bookes [. . . ] Of the proficience and advancement of learning, divine and humane. London 1605.

Baillou, Guillaume: Consiliorum medicinalium libri II. 3 vols. Ed. by Jacques Thevart. Paris 1635. 
Banzer, Marcus: Fabrica receptarum. Id est: methodus brevis, perspicua ac facilis in qua quae sint remediorum compositorum formae, quae earundem differentiae, quae componendi \& praescribendi ratio [. . . ] planissime edocetur. Augsburg 1622.

Barker-Benfield, G. J.: The culture of sensibility. Sex and society in eighteenth-century Britain. Chicago/London 1992.

Bartholinus, Thomas: De libris legendis dissertationes. Ed. by Joh. Gerh. Meuschen. Frankfurt 1711.

Bauch, Gustav: Valentin Trozendorf und die Goldberger Schule. Berlin 1921.

Bauer, Barbara: Die Rolle des Hofastrologen und Hofmathematikus als fürstlicher Berater. In: August Buck (ed.): Höfischer Humanismus. Weinheim 1989, pp. 93-117.

Bauhin, Caspar: Gynaeciorum sive de mulierum affectibus commentarii. Basel 1586.

Bayle, Ariane: Thériaque et triacleurs chez Pierre-André Mathiole. In: Sarah Voinier and Guillaume Winter (eds): Poison et antidote dans l'Europe des XVIe et XVIle siècles. Paris 2011, pp. 33-47.

Bedini, Gianni: L'orto botanico di Pisa. Piante, storia, personaggi, ruoli / The botanic garden of Pisa. Plants, history, people, roles. Pisa 2007.

Beer, Karl: Philippine Welser als Freundin der Heilkunst. In: Gesnerus 7 (1950), pp. 80-86.

Beierlein, Paul Reinhard: Der kursächsische Leibarzt Sigismund Kohlreuter (1534-1599). In: Sudhoffs Archiv 38 (1954), pp. 70-83.

Belloni Speciale, Gabriella: Falloppia, Gabriele. In: Dizionario biografico degli Italiani 44 (1994) (http://www.treccani.it/enciclopedia/gabriele-falloppia_\%28DizionarioBiografico\%29/)

Belmas, Elisabeth and Serenella Nonnis Vigilante (eds): Les relations médecin-malade des temps modernes à l'époque contemporaine. Villeneuve d'Ascq-France 2013.

Ben-Chaim, Michael: Experimental philosophy and the birth of empirical science. Boyle, Locke, and Newton. Aldershot 2004.

Benivieni, Antonio: De abditis nonnullis ac mirandis morborum et sanationum causis. Ed. by Giorgio Weber. Florence 1994.

Benzenhöfer, Udo and Wilhelm Kühlmann (eds): Heilkunde und Krankheitserfahrung in der Frühen Neuzeit. Tübingen 1992.

Bergdolt, Klaus: Das Gewissen der Medizin. Ärztliche Moral von der Antike bis heute. Munich 2004.

Berg, Alexander: Der Krankheitskomplex der Kolik-u[nd] Gebärmutterleiden in Volksmedizin und Medizingeschichte unter besonderer Berücksichtigung der Volksmedizin in Ostpreußen. Ein Beitrag zur Erforschung volkstümlicher Krankheitsvorstellungen. Berlin 1935.

Bergdolt, Klaus, Berndt Hamm and Andreas Tönnesmann (eds): Das Kind in der Renaissance. Wiesbaden 2008.

Bernardi, Francesco: Prospetto storico-critico. Dell'origine, facoltà, diversi stati, progressi, e vicende del Collegio medico chirurgico, e dell'arte chirurgica in Venezia. Venice 1797.

Berthold, Andreas: Compendium breve de terrae sigillatae usu commodissimo \& utilissimo. Sine loco 1589.

Berthold, Andreas: Nützlicher unnd nothwendiger Bericht von der Krafft, Würckung, Tugendt und Eigenschafften, der hülffreichen Terrae Sigillatae. Frankfurt 1597.

Berthold, Andreas: Terrae sigillatae nuper in Germania repertae vires atque virtutes admirandae eiusque administrandae ac usurpandae ratio. Frankfurt 1583. 
Berthold, Andreas: The wonderfull and strange effect and vertues of a new Terra sigillata lately found out in Germanie, with the right order of the applying and administring of it: being oftentimes tried and experienced. London 1587.

Bertolaso, Bartolo: La cattedra "De pulsibus et urinis" (1601-1748) nello studio padovano. In: Castalia 16 (1960), pp. 109-117.

Bertolaso, Bartolo: Richerche d'archivio su alcuni aspetti dell'insegnamento medico presso la Università di Padova nel cinque- e seicento. In: Acta medicae historiae patavina 6 (1958-59), pp. 17-37.

Beukers, Harm: Clinical teaching in Leiden from its beginning until the end of the eighteenth century. In: idem and J. Moll (eds): Clinical teaching, past and present. Amsterdam/ Atlanta, GA, pp. 139-152.

Bianchi, Massimo Luigi: Il tema dell'esperienza in Paracelso. In: Marco Veneziani (ed.): Experientia. Florence 2002, pp. 199-216.

Bianchi, Massimo Luigi: Occulto e manifesto in Jean Fernel e Pietro Severino. In: Atti e memorie dell'Accademia toscana di scienze e lettere “La Colombaria” 47 (1982), pp. 185-248.

Bienert, Karl J.: Böhm[isch] Leipa, das Verkehrs-, Wirtschafts- und Kulturzentrum Böhmens. In: Heimat-Buch. Bodenbach a. Elbe [c. 1937], pp. 1-5.

Bigotti, Fabrizio: Physiology of the soul. Mind, body and matter in the Galenic tradition of the late Renaissance (1550-1630). Turnhout 2019.

Biow, Douglas: The culture of cleanliness in Renaissance Italy. Ithaca/London 2006.

Blair, Ann: Humanist methods in natural philosophy. The commonplace book. In: Journal of the history of ideas 53 (1992), pp. 541-551.

Blair, Ann: Reading strategies for coping with information overload, ca. 1550-1700. In: Journal of the history of ideas 64 (2003), pp. 11-28.

Blair, Ann M.: Too much to know. Managing scholarly information before the modern age. New Haven/London 2010.

Bloch, Iwan: Der Ursprung der Syphilis. Eine medizinische und kulturgeschichtliche Untersuchung. 2 vols. Jena 1901/1911.

Bodenstein, Adam von (ed.): Metamorphosis. Basel 1572.

Bodin, Jean: Universae naturae theatrum: in quo rerum omnium effectrices causae et fines contemplantur et continuae series quinque libris discutiuntur. Lyon 1596.

Boehm, Laetitia u.a. (ed.): Biographisches Lexikon der Ludwig-Maximilians-Universität München. Part 1: Ingolstadt-Landshut 1472-1826. Berlin 1998.

Boissier de Sauvages, François: Nosologia methodica sistens morborum classes juxta Sydenhami mentem et botanicorum ordinem. Vol. 1. Venice 1773.

Bonnet, Théophile: Sepulchretum sive anatomia practica ex cadaveribus morbo denatis. Geneva 1679.

Bösch, Alexander: Liber familiarium personalium, das ist, Verzeichnus waß sich mit mir, und der meinigen in meiner haußhaltung, sonderliches begeben und zugetragen hatt. Lebensbericht und Familiengeschichte des Toggenburger Pfarrers Alexander Bösch (1618-1693). Ed. by Lorenz Heiligensetzer. Basel 2001.

Boscius, Joannis Loneus: De lapidibus qui nascuntur in corpore humano, et praecipue renibus ac vesica, et ipsorum curatione theses. Respondente Andrea Helepyro. Ingolstadt 1580.

Botalli, Leonardo: Commentarioli duo, alter de medici, alter de aegroti munere. Lyon 1565.

Bottoni, Albertino: De morbis muliebribus. Padua 1585. 
Boudewijns, Michael: Ventilabrum medico-theologicum, quo omnes casus cum medicos tum aegros aliosque concernentes eventilantur, et quod SS. PP. conformius, scholasticis probabilius, \& in conscientia tutius est, secernitur. Antwerp 1666

Boudon-Miller, Véronique and Guy Cobolet: Lire les médecins Grecs à la Renaissance. Paris 2004.

Bourbon, Florence: Jean Liebault (1535-1596), médicin hippocratique. Vers la gynécologie moderne. In: Renaissance and Reformation 33 (2010), pp. 61-84.

Bourdieu, Pierre: Esquisse d'une théorie de la pratique, précédé de trois études d'ethnologie kabyle. Geneva 1972.

Bourdieu, Pierre: Les trois états du capital culturel. In: Actes de la recherche en sciences sociales 30 (1979), pp. 3-6.

Bourdieu, Pierre: The forms of capital. In: John G. Richardson (ed.): Handbook of theory and research for the sociology of education. New York 1986, pp. 241-60.

Braembussche, A. A. van den: Het biografisch element in de geschiedschrijving. Een geschiedstheoretische verkenning. In: Tijdschrift voor sociale geschiedenis 15 (1989), pp. 26-60.

Brambilla, Giovanni Alessandro: Scuola Longobarda: Pavesi, Milanesi, Piemontesi, Genovesi, Piacentini, Parmigiani, Modenesi, Ferraresi, Bolognesi, Veronesi, Padovani ec. Vol. 2, 1: Secolo XVI. Milan 1781.

Brasavola, Antonio Musa: In octo libros aphorismorum Hippocratis et Galeni commentaria et annotationes. Basel 1541.

Brendecke, Arndt (ed.): Praktiken der Frühen Neuzeit. Akteure - Handlungen - Artefakte. Cologne/Weimar/Vienna 2015.

Brendel, Johann Philipp (ed.): Consilia medica celeberrimorum quorundam Germaniae medicorum. Frankfurt 1615.

Brockliss, Laurence W.B.: Curricula. In: Ridder-Symoens, History (1996), pp. 565-620.

Brockliss, Laurence W.B.: French higher education in the seventeenth and eighteenth centuries. A cultural history. Oxford 1987.

Brockliss, Laurence W.B. and Colin Jones: The medical world of early modern France. Oxford 1997.

Bröer, Ralf: Höfische Medizin. Strukturen der medizinischen Versorgung eines frühneuzeitlichen Fürstenhofes am Beispiel des Wiener Kaiserhofes (1650-1750). Habilitationsschrift. Heidelberg 2006.

Brosseder, Claudia: Im Bann der Sterne. Caspar Peucer, Philipp Melanchthon und andere Wittenberger Astrologen. Berlin 2004.

Brugi, Biagio: Gli scolari dello studio di Padova nel cinquecento. Discorso inaugurale. Padua 1903.

Brunfels, Otto: Theses seu communes loci totius rei medicae. Strasbourg 1532.

Brunschwig, Hieronymus: Buch der Cirurgia. Hantwirckung der Wund Artzney. Augsburg 1497.

Brunschwig, Hieronymus: Großes Destillierbuch. Strasbourg 1512.

Brunschwig, Hieronymus: Das Buch zu Destilliren die zusamen gethonen Ding. Strasbourg 1519.

Brunschwig, Jacques and Geoffrey E. R. Lloyd (eds), in collaboration with Pierre Pellegrin: Greek thought. A guide to classical knowledge. Cambridge/London 2000.

Buchwald, Georg: Simon Wilde aus Zwickau. Ein Wittenberger Studentenleben zur Zeit der Reformation. In: Mitteilungen der Deutschen Gesellschaft in Leipzig 9 (1894), pp. 61-111. 
Bünz, Enno: Leibärzte. In: Werner Paravicini (ed.): Höfe und Residenzen im spätmittelalterlichen Reich. Bilder und Begriffe. Vol. I: Begriffe. Ostfildern 2005, pp. 156-157.

Burckhardt, Jacob: Die Cultur der Renaissance. Ein Versuch. Basel 1860.

Burke, Peter: Images as evidence in seventeenth-century Europe. In: Journal of the history of ideas 64 (2003), pp. 273-296.

Burke, Peter: Individuality and biography in the Renaissance. In: Enno Rudolph (ed.): Die Renaissance und die Entdeckung des Individuums in der Kunst. Die Renaissance als erste Aufklärung II. Tübingen 1998, pp. 65-78.

Burke, Peter: Popular culture in early modern Europe. London 1978.

Buschmann, Nicolaus: Persönlichkeit und geschichtliche Welt. Zur praxeologischen Konzeptualisierung des Subjekts in der Geschichtswissenschaft. In: Alkemeyer, Budde and Dagmar Freist, Selbst-Bildungen (2013), pp. 125-149.

Bůžek, Václav: Ferdinand von Tirol zwischen Prag und Innsbruck. Der Adel aus den böhmischen Ländern auf dem Weg zu den Höfen der ersten Habsburger. Cologne/Weimar 2009.

Bylebyl, Jerome J.: Cardiovascular physiology in the sixteenth and early seventeenth centuries. PhD-thesis, Yale University. New Haven 1969.

Bylebyl, Jerome J.: Medicine, philosophy and humanism in Renaissance Italy. In: John W. Shirley and F. David Hoeniger (eds): Science and the arts in the Renaissance. Washington, D.C. 1985 , pp. 27-49.

Bylebyl, Jerome J.: The school of Padua: humanistic medicine in the sixteenth century. In: Charles Webster (ed.): Health, medicine and mortality in the sixteenth century. Cambridge 1979, pp. 335-370.

Bylebyl, Jerome: The manifest and the hidden in the Renaissance clinic. In: William F. Bynum and Roy Porter (eds): Medicine and the five senses. Cambridge 2004, pp. 40-60.

Calabritto, Monica: Medicina practica, consilia and the illnesses of the head in Girolamo Mercuriale and Giulio Cesare Claudini. Similarities and differences of the sexes. In: Medicina e storia 11 (2006), pp. 63-83.

Calabritto, Monica: Curing melancholia in sixteenth-century medical consilia between theory and practice. In: Medicina nei secoli 24 (2012), pp. 627-664.

Camerarius, Joachim: Arithmologia ethica, loci communes, et epigrammata. Leipzig 1552.

Capivaccia, Girolamo: De modo interrogandi aegros. In: idem: Opera omnia. Ed. by Johann Hartmann Beyer. Frankfurt 1603, pp. 236-237.

Cappelletti, Elsa M.: Le piante coltivate nell'orto botanico di Padova ai tempi di Luigi Squalermo detto Anguillara. In: Minelli, L'orto (1995), pp. 162-171.

Cardano, Girolamo: De malo recentiorum medicorum medendi usu libellus. Venice 1536.

Cardano, Girolamo: Opera quaedam lectu digna. Basel 1562.

Carlino, Andrea: Books of the body. Anatomical ritual and Renaissance learning. Chicago 1999.

Carrichter, Bartholomaeus: Kräutterbuch. Darinnen begriffen, under welchem Zeichen Zodiaci, auch in welchem Gradu ein jedes Kraut stehe, wie sie in Leib, und zu allen Schäden zu bereiten, und zu welcher Zeit sie zu colligieren sein. Strasbourg 1609.

Castelli, Bartolommeo: Lexicon medicum graecolatinum [. . .] ex Hippocrate, et Galeno desumptum. Messana 1598

Castro, Roderigo da: Medicus-politicus: sive de officiis medico-politicis tractatus. Hamburg 1614.

Castro, Roderigo da: Universa mulierum medicina. Part 2. Hamburg $1662\left(1^{\text {st }}\right.$ edn 1603). 
Cavallo, Sandra and Tessa Storey: Healthy living in late Renaissance Italy. Oxford 2014.

Celsus, Aulus Cornelius: De medicina libri octo. Ed. by Johannes Antonides van der Linden, Leiden 1657.

Celtis, Konrad: Quattuor libri amorum secundum quattuor latera Germaniae. Germania generalis. Accedunt carmina aliorum ad libros amorum pertinentia. Ed. by Felicitas Pindter. Leipzig 1934.

Champier, Symphorien: Claudii Galeni Pergameni historiales campi [. . .] in quatuor libros congesti et commentariis non poenitendis illustrati. Basel 1532.

Chauliac, Guy de: Chirurgia. Leiden 1559.

Chauliac, Guy de: Guigonis de Caulhiaco inventarium sive chirurgia magna. Ed. by Michael R. McVaugh, vol. 1: Text. Leiden 1997.

Chifflet, Jean: Singulares tam ex curationibus, quam cadaverum sectionibus observationes. Paris 1612.

Ciancio, Luca: “Per questa via s'ascende a magior seggio”. Pietro Andrea Mattioli e le scienze mediche e naturali alla corte di Bernardo Cles. In: Studi Trentini. Storia 94 (2015), pp. 159-184.

Ciancio, Luca: Many gardens - real, symbolic, visual - of Pietro Andrea Mattioli. In: Juliette Ferdinand (ed.): From art to science. Experiencing nature in the European garden 1500-1700. Treviso 2016, pp. 35-45

Cicero, Marcus Tullius: Epistulae ad familiares. Venice 1471.

Cipolla, Carlo M.: Public health and the medical profession in the Renaissance. Cambridge 1976.

Clark, Stuart: Thinking with demons. The idea of witchcraft in early modern Europe. Oxford 1997

Clauser, Christoph: Das die Betrachtung des Menschenn Harns on anderen Bericht unnütz. Sine loco [1543].

Clouse, Michele L.: Medicine, government and public health in Philip Il's Spain. Shared interests, competing authorities. Farnham 2011.

Codronchi, Baptista: De christiana ac tuta medendi ratione. Ferrara 1591.

Coiter, Volcher: Externarum et internarum principalium humani corporis partium tabulae, atque anatomicae exercitationes observationesque variae. Nürnberg 1573.

Collinus, Matthaeus et alii: Prima farrago sacri argumenti poematum ab aliquot studiosis poeticae bohemis scriptorum diversis temporibus ad nobilem et clarissimum virum

D. loannem Seniorem Hoddeiovinum ad Hoddeiova. Prague [1561].

Collinus, Matthaeus et alii: Tertia farrago poematum. Prague 1561.

Collinus, Matthaeus et alii: Quarta farrago poematum. Prague 1562.

Colombo, Realdo: De re anatomica libri XV. Venice 1559.

Comparetti, Andrea: Saggio della Scuola Clinica nello Spedale di Padova. Padua 1793.

Cook, Harold J.: Good advice and little medicine. The professional authority of early modern English physicians. In: Journal of British studies 33 (1994), pp. 1-31.

Cook, Harold J.: Medicine. In: Katharine Park and Lorraine Daston (eds): Early modern science (= The Cambridge History of Science, vol. 3). Cambridge 2006, pp. 407-434.

Cook, Harold J.: Physicians and natural history. In: Nicholas Jardine, James Secord and Emma Spary (eds): Cultures of natural history. Cambridge 1996, pp. 91-105.

Cook, Harold J.: Trials of an ordinary doctor: Joannes Groenevelt in seventeenth-century London. Baltimore 1994. 
Cook, Harold J.: Victories for empiricism, failures for theory. Medicine and science in the seventeenth-century. In: Charles T. Wolfe and Ofer Gal (eds): The body as object and instrument of knowledge. Embodied empiricism and early modern science. Dordrecht 2010, pp. 9-32.

Cooper, Glen M.: Approaches to the critical days in late medieval and Renaissance thinkers. In: Early science and medicine 18 (2013), pp 536-565.

Cordus, Euricius: De urinis. Das ist von rechter Besichtigunge des Harns und ihrem Mißbrauch. Ed. by J. Dryander. Frankfurt 1543.

Cordus, Valerius: Pharmacorum omnium, quae quidem in usu sunt, conficiendorum ratio: vulgo vocant dispensatorium pharmacopolarum. Nürnberg 1546.

Cornarius, Janus: Medicina, sive medicus, liber unus. Eiusdem orationes II: I. Hippocrates, sive doctor verus: II. de rectis medicinae studiis amplectendis. Basel 1556.

Cornaro, Alvise: Discorsi della vita sobria. Milan 1627.

Crato, Johannes: Consiliorum et epistolarum medicinalium liber. Ed. by Lorenz Scholz. Frankfurt am Main 1591.

Crato, Johannes: Consiliorum et epistolarum medicinalium liber quintus. Hanau 1594.

Cunsolo, Elisabetta: Giulio Casserio e la pubblicazione del De Vocis Auditusque Organis tra Padova e Ferrara all'inizio del '600. In: Mélanges de l'école française de Rome 120-122 (2008), pp. 385-405.

Da Monte, Giovanni Battista: Consilia medica omnia, quae ullibi extant, partim antea, partim nunc primum edita. Ed. by Girolamo Donzellini. Nürnberg 1559.

Da Monte, Giovanni Battista: Consultationum medicinalium centuria prima. Ed. by Valentinus Lublinus. Venice 1554 (further edn 1556).

Da Monte, Giovanni Battista: Consultationum medicinalium ad varia morborum genera, centuria tertia. Venice 1558.

Da Monte, Giovanni Battista: Consultationum medicinalium centuria secunda. Venice 1559.

Da Monte, Giovanni Battista: Consultationum medicarum opus absolutissimum. Ed. by Johannes Crato. Basel 1565.

Da Monte, Giovanni Battista: De uterinis affectibus. In: idem: Opuscula. Ed. by Valentinus Lublinus. Venice 1554, foll. 63r-109v.

Da Monte, Giovanni Battista: Lectiones de urinis. Ed. by Franz Emmerich. Vienna 1552.

Da Monte, Giovanni Battista: Methodus de elementis, cui accessit De syphillidos lue tractatus, unacum regulari cura huius morbi Benedicti faventini. Vienna 1553.

Da Monte, Giovanni Battista: Opuscula varia ac praeclara. 2 vols. Basel 1558.

Darmon, Pierre: Le tribunal de l'impuissance. Paris 1979.

Daston, Lorraine: Perché i fatti sono brevi? In: Quaderni storici 108 (2001), pp. 745-770.

Daston, Lorraine: Taking note(s). In: Isis 95 (2004), pp. 443-448.

Daston, Lorraine and Katharine Park: Wonders and the order of nature, 1150-1750. New York 1998.

Davis, Dona Lee and Richard G. Whitten: Medical and popular traditions of nerves. In: Social science and medicine 26 (1988), pp. 1209-1222.

Davis, Dona Lee and Setha M. Low (eds): Gender, health, and illness. The case of nerves. New York 1989.

De Renzi, Salvatore (ed.): Collectio salernitana. 5 vols. Naples 1852-1859.

De Renzi, Silvia: A career in manuscripts: Genres and purposes of a physician's writing in Rome, 1600-1630. In: Italian studies 66 (2011), pp. 234-248. 
Dear, Peter: The meanings of experience. In: Katharine Park and Lorraine Daston (eds): Early modern science (= The Cambridge History of Science, vol. 3). Cambridge 2006, pp. 106-131.

Debru, Armelle: Galen. In: Brunschwig/Lloyd,Greek thought (2000), pp. 618-630.

Debus, Allen G.: The French Paracelsians. The chemical challenge to medical and scientific tradition in early modern France. Cambridge 1991.

Delisle, Candice: The letter: Private of public place? The Mattioli-Gesner controversy about the aconitum primum. In: Gesnerus 61 (2004), pp. 161-176.

Dell'Acqua, Gioan Battista: Giovanni Manardo medico e clinico. In: Atti del convegno internazionale per la celebrazione del V centenario della nascita di Giovanni Manardo 1462-1536. Ferrara 1963, pp.8-42.

Demaitre, Luke: Medieval medicine. The art of healing from head to toe. Santa Barbara/ Denver/Oxford 2013.

Demaitre, Luke: Medieval notions of cancer. Malignancy and metaphor. In: Bulletin of the history of medicine 72 (1998), pp. 609-637.

Demaitre, Luke: Straws in the wind. Latin writings on asthma between Galen and Cardano. In: Allergy and asthma proceedings 23 (2002), pp. 61-93.

Dinges, Martin, Kay Peter Jankrift, Sabine Schlegelmilch and Michael Stolberg (eds): Medical practice, 1600-1900. Physicians and their patients. Leiden 2016.

Donati, Marcello: De medica historia mirabili libri sex. Venice 1588.

Dodoens, Rembert: Medicinalium observationum exempla rara, recognita et aucta. Cologne 1581.

Dondi, Raffaele Flaminio: Elideo Padovani. Medico forlivese del secolo XVI. In: Atti e memorie dell'Accademia di storia dell'arte sanitaria 117 (1951), pp. 139-144.

Dondi, Raffaele Flaminio: Cenni sul medico forlivese Alessandro Padovani (?-1637) e sulla sua biblioteca. In: Rivista di storia della medicina 19 (1975), pp. 190-198.

Dotzauer, Winfried: Deutsches Studium und deutsche Studenten an europäischen Hochschulen (Frankreich, Italien) und die nachfolgende Tätigkeit in Staat, Kirche und Territorium in Deutschland. In: Erich Maschke and Jürgen Sydow (eds): Stadt und Universität im Mittelalter und in der frühen Neuzeit. Tübingen 1974, pp. 112-141.

Douglas, Mary: Purity and danger. An analysis of concepts of pollution and taboo. London 1978.

Drembach, Martin von: De atra bile disputatio medica. Resp. Blasius Thammüller. [Leipzig]: [1548].

Drexel, Jeremias: Aurifodina artium et scientiarum omnium excerpendi solertia. Munich 1638.

Duden, Barbara: Geschichte unter der Haut. Ein Eisenacher Arzt und seine Patientinnen um 1730. Stuttgart 1987.

Dulieu, Louis: Félix Platter, étudiant de l’École de médecine de Montpellier. In: Ulrich Tröhler (ed.): Felix Platter (1536-1614) in seiner Zeit. Basel 1991, pp.17-20.

Dulieu, Louis: Guillaume Rondelet. In: Clio medica 1 (1966), pp. 89-111.

Dulieu, Louis: La médecine à Montpellier. Vol. 2: La Renaissance. Avignon 1979.

Dunus, Thaddaeus: Muliebrium morborum omnis generis remedia. Strasbourg 1565.

Durling, Richard J.: A chronological census of Renaissance editions and translations of Galen In: Journal of the Warburg and Courtauld Institutes 24 (1961), pp. 230-305.

Durling, Richard J.: Conrad Gesner's “Liber amicorum” 1555-1565. In: Gesnerus 22 (1965), pp. 134-159. 
Durling, Richard J.: Girolamo Mercuriale's De modo studendi. In: Osiris N. S. 6 (1991), pp. 181-195.

Dumaître, Paule: Ambroise Paré. Chirurgien de quatre rois de France. Paris 1986.

Eadie, Mervyn J. and Peter F. Bladin: A disease once sacred. A history of the medical understanding of epilepsy. Eastleigh 2001.

Eamon, William: How to read a book of secrets. In: Elaine Leong and Alisha Rankin (eds): Secrets and knowledge in medicine and science, 1500-1800. Farnham 2011, pp. 23-46.

Eamon, William: Science and the secrets of nature. Books of secrets in medieval and early modern culture. Princeton 1994.

Ebelová, Ivana (ed.): Pamětní kniha města české Lípy. Ústí nad Labem 2005.

Eckart, Wolfgang U.: Anmerkungen zur "Medicus politicus"- und "Machiavellus Medicus"Literatur des 17. und 18. Jahrhunderts. In: Benzenhöfer/Kühlmann, Heilkunde (1992), pp. 114-129.

Eisenberg, Leon: The physician as interpreter. Ascribing meaning to the illness experience. In: Comprehensive psychiatry 22 (1981), pp. 239-248.

Elias, Norbert: Über den Prozeß der Zivilisation. Soziogenetische und psychogenetische Untersuchungen. 2 vols. $6^{\text {th }}$ edn. Frankfurt 1979.

Elkeles, Barbara: Arzt und Patient in der medizinischen Standesliteratur der Frühen Neuzeit. In: Benzenhöfer/Kühlmann, Heilkunde (1992), pp. 131-143.

Elkeles, Barbara: Medicus und Medikaster: Zum Konflikt zwischen akademischer und “empirischer" Medizin im 17. und frühen 18. Jahrhundert. In: Medizinhistorisches Journal 22 (1987), pp. 197-211.

D'Elvert, Christian: Zur Geschichte der Pflege der Naturwissenschaften in Mähren und Schlesien, insbesondere der Naturkunde dieser Länder, mit Rücksicht auf Böhmen und Österreich. Brünn 1868.

Erastus, Thomas: De lamiis seu strigibus. Basel 1578.

Erastus, Thomas: De medicina nova Philippi Paracelsi. 4 parts. Basel 1572-1573.

Erastus, Thomas: Disputationum et epistolarum medicinalium volumen doctissimum. Ed. by Theophilus Maderus. Zürich 1595.

Elkeles, Barbara: Arzt und Patient in der medizinischen Standesliteratur der Frühen Neuzeit. In: Benzenhöfer/Kühlmann, Heilkunde (1992), pp. 131-143.

Ellenbog, Nikolaus: Briefwechsel. Ed. by Andreas Bigelmair and Friedrich Zoepfl. Münster 1938.

Enenkel, Karl A. E.: Die Grundlegung humanistischer Selbstpräsentation im Brief-Corpus: Francesco Petrarcas Familiarium rerum libri XXIV. In: van Houdt, Self-presentation (2002), pp. 367-384.

Enenkel, Karl A. E.: In search of fame. Self-representation in neo-Latin humanism. In: Stephen Gersh and Bert Roest (eds): Medieval and Renaissance humanism: Rhetoric, representation and reform. Leiden 2003, pp. 93-113.

Erasmus, Desiderius: De conscribendis epistolis. Cambridge 1521.

Erasmus, Desiderius: De duplici copia verborum ac rerum commentarii duo. Paris 1514.

Ettmüller, Michael: Opera omnia theoretica et practica. Part 2. Lyon 1685.

Evans, Jennifer and Sara Read: Maladies and medicine. Exploring health and healing 1540-1740. Barnsley 2017.

Fabiani, Giuseppe: La vita di Pietro Andrea Mattioli. Ed. by Luciano Bianchi. Siena 1872.

Fabricius, Wilhelm: Opera omnia quae extant. Frankfurt 1646. 
Fabricius, Wilhelm: Wund-Artzney. Gantzes Werck, und aller Bücher, so viel deren vorhanden. Frankfurt 1652.

Facciolati, Jacobus: Fasti Gymnasii Patavini [. . . ] collecti ab anno MDXVII quo restitutae scholae sunt ad MDCCLVI. Padua 1757.

Falloppia, Gabrielle: De cauteriis tractatus. Appended to idem: Tractatus de compositione medicamentorum dilucidissimus. Venice 1570 , foll. 65r-72v.

Falloppia, Gabrielle: De humani corporis anatome compendium. Venice 1571.

Falloppia, Gabrielle: De medicatis aquis atque de fossilibus. Venice 1564.

Falloppia, Gabrielle: De tumoribus praeter naturam. In: idem: Opera genuina omnia. Vol 3. Venice 1606, foll. 1r-109v.

Falloppia, Gabrielle: Expositio in librum Galeni de ossibus. Huic accesserunt observationes anatomicae eiusdem authoris. Ed. by F. Michinus. Venice 1570.

Falloppia, Gabrielle: Observationes anatomicae ad Petrum Mannam. Venice 1561.

Falloppia, Gabrielle: Tractatus de decoratione. In: idem: Opuscula. Part 2. Padua 1566, foll. $34 \mathrm{r}-51 \mathrm{v}$

Fantuzzi, Giovanni: Notizie degli scrittori bolognesi. Vol. 6. Bologna 1788.

Fausti, Daniela (ed.): La complessa scienza dei semplici. Atti delle celebrazioni per il V centenario della nascita di Pietro Andrea Mattioli, Siena, 12 marzo-19 novembre. Siena 2001.

Favaro, Antonio (ed.): Atti della Nazione Germanica Artista nello Studio di Padova. Vol. 1. Venice 1911.

Favaro, Giuseppe: Contributi alle biografia di Girolamo Fabrici d'Acquapendente. In: Memorie e documenti per la storia della Università di Padova. Vol. 1. Padua 1922, pp. 241-348.

Favaro, Giuseppe: Gabrielle Falloppa modenese (MDXXII-MDLXII). Studio biografico. Modena 1928.

Faventinus, Leonellus de Victoriis: De aegritudinibus infantium tractatus admodum salutifer. Venice 1557.

Fernel, Jean: Consiliorum liber. Cui accesserunt responsa quaedam clarorum medicorum Parisiensium. In: idem: Universa medicina. Geneva 1644, pp. 247-397 (appended to De abditis rerum).

Fernel, Jean: De abditis rerum causis libri duo. In: Fernel, Universa medicina (1644) (separate pagination).

Fernel, Jean: Universa medicina. Geneva 1542.

Fernel, Jean, Universa medicina. Geneva 1644.

Ferrarius, Omnibonus: De arte medica infantium quorum duo priores de tuenda eorum sanitate, posteriores de curandis morbis agunt. Brixen 1577.

Ferretto, Silvia: Bassiano Lando e la "scienza" della medicina tra filosofia e teologia nel XVI secolo. Tesi, Università degli Studi di Trento, ciclo XXII, 2006-2009.

Ferretto, Silvia: Maestri per il metodo di trattar le cose Bassiano Lando, Giovan Battista da Monte e la scienza della medicina nel XVI secolo. Padua 2012.

Ferri, Sara (ed.): Pietro Andrea Mattioli, Siena 1501-Trento 1578. La vita, le opere. Perugia 1997.

Ferri, Sara: Il “Dioscoride”, i “Discorsi”, i “Commentarii”: Gli amici e i nemici. In: eadem, Mattioli (1997), pp. 15-48.

Feustel, Robert: Grenzgänge. Kulturen des Rauschs seit der Renaissance. Munich 2013.

Fichtner, Gerhard: Padova e Tübingen: La formazione medica nei secoli XVI e XVII. In: Acta medicae historiae patavina 19 (1972-73), pp. 43-62.

Ficino, Marsilio: De triplici vita: libri tres. Venice 1498 (repr. Hildesheim 1978). 
Findlen, Paula and Pamela H. Smith (eds): Merchants and marvels. Commerce and the representation of nature in early modern Europe. New York 2002.

Findlen, Paula: Possessing nature. Museums, collecting, and scientific culture in early modern Italy. Berkeley/Los Angeles/London 1994.

Findlen, Paula: The formation of a scientific community. Natural history in sixteenth-century Italy. In: Anthony Grafton and Nancy Siraisi (eds): Natural particulars. Nature and the disciplines in Renaissance Europe. Cambridge, MA, 1999, pp. 369-400.

Findlen, Paula: The death of a naturalist: Knowledge and community in Renaissance Italy. In: Gideon Manning and Cynthia Klestinec (eds): Professors, physicians and practices in the history of medicine: Essays honoring Nancy Siraisi. New York 2017, pp. 127-167.

Finkler, Kaja: The universality of nerves. In: Davis/Low, Gender (1989), pp. 169-179.

Finucci, Valeria: The prince's body. Vincenzo Gonzaga and Renaissance medicine. Cambridge/ London 2015.

Finzel, Hiob: Wunderzeichen. Warhafftige Beschreybung und gründlich Verzeichnuß schröcklicher wunderzeichen und Geschichten, die von dem Jar an M. D. XVII bis auff yetziges Jar M.D.LVI geschehen und ergangen sindt nach der Jarzal. Nürnberg 1556.

Fischer, Klaus: Hartmann Schedel in Nördlingen. Das pharmazeutisch-soziale Profil eines spätmittelalterlichen Stadtarztes. Würzburg 1996.

Fischer-Homberger, Esther: Hypochondrie. Melancholie bis Neurose. Krankheiten und Zustandsbilder. Bern 1970.

Fissell, Mary: Vernacular bodies. The politics of reproduction in early modern England. Oxford 2004.

Flamm, Heinz: Bader - Wundarzt - Medicus. In: idem and Karl Mazakarini (eds): Bader Wundarzt - Medicus. Heilkunst in Klosterneuburg. Begleitpublikation zur Ausstellung. Klosterneuburg 1996, pp. 7-40.

Fonseca, Rodericus: Opusculum, quo adolescentes ad medicinam facile capessendam instruuntur, casus omnium febrium methodice discutiuntur, \& curantur. Florence 1596.

Forcher, Michael: Erzherzog Ferdinand II. Landesfürst von Tirol. Sein Leben. Seine Herrschaft. Sein Land. Innsbruck/Vienna 2017.

Foreest, Pieter van: Observationum et curationum medicinalium libri XXXII. Leiden 1603-1606.

Foreest, Pieter van: Observationum et curationum chirurgicarum libri quatuor posteriores, de vulneribus, ulceribus, fracturis, luxationibus. Leiden 1601.

Foreest, Pieter van: Uromanteia. Das ist warhafftiger und wolgegründter Bericht von den vielfaltigen Urtheilen unnd Weissagungen auß den Urinen oder Wassern. Frankfurt 1620.

Foreest, Pieter van: Observationum et curationum medicinalium ac chirurgicarum opera omnia. Frankfurt 1634.

Fortuna, Stefania: The Latin editions of Galen's Opera omnia (1490-1625) and their prefaces. In: Early science and medicine 17 (2012), pp. 391-412.

Fossati, Pier Maria (ed.): Girolamo Fabrizi da Acquapendente. Medico e anatomista. La vita e le opere. Note in margine alla mostra. Acquapendente 1988.

Fracanzano, Antonio: De morbo gallico fragmenta quaedam elegantissima, ex lectionibus anni MDLXII Bononiae. Appended to Gabrielle Falloppi, De morbo gallico liber. Venice 1574, pp. 186-219.

Fracanzano, Antonio: De morbo gallico liber. Ed. by Camillo Cochio. Bologna 1564.

Fracastoro, Girolamo: Syphilis sive morbus gallicus. Verona 1536.

French, Roger: Medicine before science. The rational and learned doctor from the Middle Ages to the Enlightenment. Cambridge 2003. 
Friedrich, Udo: Naturgeschichte zwischen artes liberales und frühneuzeitlicher Wissenschaft. Conrad Gessners “Historia animalium” und ihre volkssprachige Rezeption. Tübingen 1995.

Friedrich IV. von der Pfalz: Das Tagebuch und Ausgabenbuch des Churfürsten Friedrich IV. von der Pfalz. Ed. by J. Wille. In: Zeitschrift für die Geschichte des Oberrheins 33 (1880), pp. 201-295.

Frijhoff, Willem: Patterns. In: Ridder-Symoens, History (1996), pp. 43-105.

Fuchs, Leonhard: De historia stirpium commentarij insignes. Basel 1542.

Fučíková, Eliška et alii (eds): Rudolf Il and Prague. The court and the city. London 1997.

Fürst, Susanne: Das Arztporträt in der Frühen Neuzeit. Diss. med. Regensburg 2009.

Galen: De morborum \& symptomatum differentijs \& causis libri 6. Transl. by Wilhelm Copus. Lyon 1547.

Galen: Opera omnia. 20 vols. Ed. by C. G. Kühn. Leipzig 1822 (repr. Hildesheim 1964).

Galen: Quod optimus medicus sit quoque philosophus. Transl. by Desiderius Erasmus. In: idem: Protreptikos logos pros tas technas [. . .]. Ad artes exhortatio. De optima doctrina. Quod optimus medicus sit quoque philosophus. Paris 1547, pp. 27-31.

Gallo, Andrea: Fascis de peste, peripneumonia pestilentiali cum sputo sanguinis, febre pestilentiali, ac quibusdam symptomatibus, in quinque fasciculos digestus. Brixen 1567.

Gasser, Achilles Pirmin: Ainfeltiger und gegrünter Bericht, wie menigklich sich in pestilentzischem Ubergang, mit Artznyen, und anderer Lybsnot, halten, beweren und genören soll. Nürnberg 1544.

Gasser, Achilles Pirmin: Catalogus regum omnium, quorum sub christiana professione per Europam adhuc regna florent. Augsburg 1552.

Gasser, Achilles Pirmin: De regibus Hierosolymitanis. Basel 1555.

Gasser, Achilles Pirmin: Historiarum et chronicorum mundi epitome velut index. Basel 1532.

Gaudin, Léon (ed.): Félix et Thomas Platter à Montpellier, 1552-1559, 1595-1599. Notes de voyage de deux étudiants balois. Montpellier 1892.

Gentilcore, David: Food and health in early modern Europe. Diet, medicine and society, 1450-1800. London 2016.

Gentilcore, David: Healers and healing in early modern Italy. Manchester 1998.

Gentilcore, David: Was there a "popular medicine" in early modern Europe? In: Folklore 115 (2004), pp. 151-166.

Germain, Alexandre Charles: La médecine arabe et la médecine grecque à Montpellier. In: idem (ed.): Mélanges académiques d'histoire et d'archéologie. Vol. 5. Montpellier 1877 (separate pagination).

Germain, Alexandre Charles: Les anciennes thèses de l'École de médecine de Montpellier. Collation des grades et concours professoraux. Montpellier 1886.

Germain, Alexandre Charles: Les étudiants de l'École de médecine de Montpellier au XVIe siècle. Étude historique sur le Liber procuratoris studiosorum. Paris 1876.

Germain, Alexandre Charles: Les pèlerins de la science à Montpellier. In: Bulletin de la Société Languedocienne de Géographie 1 (1878), pp. 161-181.

Gersdorff, Hans von: Feldtbuch der Wundartzney. Strasbourg 1517.

Gessner, Conrad: Historia animalium. Zürich 1551-1558 and 1587.

Gessner, Conrad, Pandectae sive partitionum universalium [. . .] libri XXI. Zürich 1548.

Gessner, Conrad: Sanitatis tuendae praecepta: cum aliis, tum literarum studiosis hominibus, \& iis qui minus exercentur, cognitu necessaria; contra luxum conviviorum; contra notas astrologicas ephemeridum de secandis venis. Zürich 1556. 
Gillet, Johann Franz Albert: Crato von Crafftheim und seine Freunde. Ein Beitrag zur Kirchengeschichte. 2 parts. Frankfurt am Main 1860.

Glaubitz, Michael von: Zwo Haußtaffeln und Underricht fur die Reichen und Armen zur Sommer und Winterzeit wider die fürstehende schrecklich und wegfressende Pestilentz. Mainz 1584.

Glück, Helmut: Deutsch als Fremdsprache in Europa vom Mittelalter bis zur Barockzeit. Berlin/ New York 2002.

Good, Byron J.: Medicine, rationality and experience. An anthropological perspective. Cambridge 1994.

Gößwein, Elisabeth: Mater puerorum. Das epileptische Kind im Fokus ärztlicher Fallberichte der Frühen Neuzeit. Diss. med. Univ. Regensburg 2016.

Graf-Stuhlhofer, Franz: Humanismus zwischen Hof und Universität. Georg Tannstetter (Collimitius) und sein wissenschaftliches Umfeld im Wien des frühen 16. Jahrhunderts. Vienna 1996.

Grafton, Anthony: Cardano's cosmos. The worlds and works of a Renaissance astrologer. Cambridge, MA 1999.

Green, Monica: Women's medical practice and health care in medieval Europe. In: Signs 14 (1989), pp. 434-473.

Green, Monica: Making women's medicine masculine: The rise of male authority in pre-modern gynecology. Oxford 2008.

Greenblatt, Stephen: Renaissance self-fashioning. From More to Shakespeare. Chicago/ London 1980.

Greiner, J.: Dinkelsbühler Arzt-Instruktionen von 1556. In: Sudhoffs Archiv 28 (1935), pp. 123-125.

Grell, Ole Peter: Conflicting duties: Plague and the obligations of early modern physicians towards patients and commonwealth in England and the Netherlands. In: Andrew Wear, Johanna Geyer-Kordesch and Roger French (eds): Doctors and ethics: The earlier historical setting of professional ethics. Amsterdam 1993, pp. 131-152.

Grell, Ole Peter (ed.): Paracelsus: The man and his reputation, his ideas and their transformation. Leiden 1998.

Grendler, Paul F.: The universities of the Italian Renaissance. Baltimore 2002.

Grodzynski, Denise: Superstitio. In: Revue des études anciennes 76 (1974), pp. 36-60.

Groß, Dominik and Jan Steinmetzer: Strategien ärztlicher Selbstautorisierung in der frühneuzeitlichen Medizin. Das Beispiel Volcher Coiters (1534-1576). In:

Medizinhistorisches Journal 40 (2005), pp. 275-320.

Größing, Sigrid-Maria: Kaufmannstochter im Kaiserhaus. Philippine Welser und ihre Heilkunst. [Vienna] 1992.

Größing, Sigrid-Maria: Die Heilkunst der Philippine Welser, Außenseiterin im Hause Habsburg. Augsburg 1998.

Gryll, Lorenz: Oratio de peregrinatione studii medicinalis ergo suscepta. [Ingolstadt] 1566.

Guarino, Mauro: Profilo storico degli ospedali di Bologna e Ferrara. In: Graziano Campanini, Mauro Guarino and G. Lippi (eds): Le arti della salute. Il patrimonio culturale e scientifico della sanità pubblica in Emilia-Romagna. Milan 2005, pp.77-93.

Guidi, Guido: De curatione generatim, in: idem: Opera omnia sive ars medicinalis. Frankfurt 1626 (separate pagination).

Gunnoe, Charles: The debate between Johann Weyer and Thomas Erastus on the punishment of witches. In: James van Horn Melton (ed.): Cultures of communication from Reformation 
to Enlightenment. Constructing publics in the early modern German lands. Aldershot 2002, pp. 257-285.

Gunnoe, Charles D.: Thomas Erastus and his circle of anti-Paracelsians. In: Joachim Telle (eds): Analecta paracelsica. Studien zum Nachleben Theophrast von Hohenheims im deutschen Kulturgebiet der frühen Neuzeit. Stuttgart 1994, pp.127-148.

Guth, Gustav: Das Idyll von den Teplitzer Heilquellen (Idyllion de thermis teplicensibus) des Thomas Mitis (1550). In: Erzgebirgszeitung 51, nº 9 (1930), pp. 125-129, 142-145 and 161-163.

Haag, Sabine and Sandbichler, Veronika (eds): Ferdinand II. 450 Jahre Tiroler Landesfürst. Innsbruck 2017.

Hacke, Daniela: Von der Wirkungsmächtigkeit des Heiligen: Magische Liebeszauberpraktiken und die religiöse Mentalität venezianischer Laien in der frühen Neuzeit. In: Historische Anthropologie 3 (2001), pp. 311-332.

Handsch, Georg: Ad lectorem. In: Petrus Sibyllenus: De peste liber. Prague 1564.

Handsch, Georg: Calendarium novum rythmicis sententiis apposite ad unumquodque tempus vel festum accomodatis concinnatum. In: Matthaeus Collinus: Elementarius libellus in lingua latina \& boiemica pro novellis scholasticis. Prague 1550.

Handsch, Georg: Die Elbefischerei in Böhmen und Meißen. Ed. by Ottokar Schubert. Prague 1933.

Handsch, Georg: [Two letters to Simon Ennius and a letter to Matthaeus Collinus]. In: Časopis Musea království českého 87 (1913), pp. 167-169 and p. 179.

Handsch, Georg: In effigiem reginae Mariae. In: Thomas Mitis (ed.): In felicem inaugurationem sereniss[imi] regis Maximiliani, \& sereniss[imae] reginae Mariae, chorus davidicus. Prague 1562.

Handsch, Georg: In icona R[egis] Maximiliani. In: Thomas Mitis (ed.): In felicem inaugurationem sereniss[imi] regis Maximiliani, \& sereniss[imae] reginae Mariae, chorus davidicus. Prague 1562.

Handsch, Georg: Dedicatory poem. In: Wenzel Nicolaides (ed.): Cantiones evangelicae ad usitatas harmonias, quae in ecclesiis boemicis per totius anni circulum canuntur, accomodatae, praecipua Christi beneficia breviter complectentes. Wittenberg 1554.

Handsch, Georg: Zum Leser. In: Mattioli, Kreutterbuch (1563).

Handsch, Georg (ed.): Secunda farrago elegiarum et idylliorum ab aliquot studiosis poeticae bohemis scriptorum diversis temporibus ad nobilem et clarissimum virum D. Ioannem Seniorem Hoddeiovinum ad Hoddeiova. Prague 1561.

Hankinson, Robert J.: The Cambridge companion to Galen. Cambridge 2008.

Hantschel, Franz: Heimatkunde des politischen Bezirkes B.-Leipa. Böhmisch Leipa 1911.

Hart, James: The arraignment of urines, wherein are set downe the manifold errors and abuses of ignorant urine-monging [sic!] empirickes, cozening quacksalvers, women-physitians and the like stuffe. London 1623.

Harvey, William: Exercitatio anatomica de motu cordis et sanguinis in animalibus. Frankfurt 1628.

Hase, Eduard Friedrich: Dr. Thomas Reinesius, Stadtphysikus und Bürgermeister zu Altenburg. Ein Lebensbild aus dem 17. Jahrhundert. In: Mitteilungen der Geschichts- und Altertumsforschenden Gesellschaft des Osterlandes 4 (1858), pp. 309-348.

Hasse, Hans Peter and Günther Wartenberg (eds): Caspar Peucer 1525-1602. Wissenschaft, Glaube und Politik im konfessionellen Zeitalter. Leipzig 2004. 
Havenreuter, Johann Ludwig: Theses medicae de iis rebus quae in principio artis medicae Galeni traduntur. Resp. Joh. Sebastian Frid. Strasbourg 1568.

Heide, Anton de: Vertoog over de onzekerheit der piskijkerij en bedrieglijkheit der piskijkeren. Amsterdam 1682.

Heigel, Karl Theodor von: Schrenck von Notzing. In: Allgemeine Deutsche Biographie 32 (1891), pp. 485-488.

Hechstetter, Philippus: Rararum observationum medicinalium decades tres. Augsburg 1624.

Heischkel, Edith: Die Welt des praktischen Arztes. In: Walter Artelt and Walter Rüegg (eds): Der Arzt und der Kranke in der Gesellschaft des 19. Jahrhunderts. Frankfurt 1967, pp. 1-16.

Hejnic, Josef: Dva humanisté v roce 1547 (Jan Šentygar a Bohuslav Hodějovský). Prague 1957.

Hejnová, Miroslava: Pietro Andrea Mattioli 1501-1578. U př́ležitosti 500. výroči narození/In occasione del V. centenario della nascita. Prague 2001.

Helm, Jürgen: Die Galenrezeption in Philipp Melanchthons De anima (1540/1552). In: Medizinhistorisches Journal 31 (1996), pp. 298-321.

Helm, Jürgen: Zwischen Aristotelismus, Protestantismus und zeitgenössischer Medizin. Philipp Melanchthons Lehrbuch “De anima” (1540/1552). In: Jürgen Leonhardt (ed.): Melanchthon und das Lehrbuch des 16. Jahrhunderts. Rostock 1997, pp. 175-191.

Helman, Cecil G.: Culture, health and illness. $5^{\text {th }}$ edn. Boca Raton 2007.

Helman, Cecil G.: "Feed a cold, starve a fever". Folk models of infection in an English suburban community and their relation to medical treatment. In: Culture, medicine and psychiatry 2 (1978), pp. 107-137.

Helmich, Egon: Die Briefe Konrad Gesners an Crato von Krafftheim nach der Briefsammlung von 1566. Diss. med. Düsseldorf 1938.

Henderson, John: The Renaissance hospital. Healing the body and healing the soul. New Haven 2006.

Henkel, Arthur and Albrecht Schöne (eds): Emblemata. Handbuch zur Sinnbildkunst des XVI. und XVII. Jahrhunderts. Stuttgart/Weimar 1996.

Herbst, Klaus-Dieter: Biobibliographisches Handbuch der Kalendermacher von 1550 bis 1750 (https://www.presseforschung.uni-bremen.de/dokuwiki/doku.php?id=startseite).

Herbst, Klaus-Dieter: Der Arzt als Autor von Jahreskalendern. In: Salatowsky/Stolberg, Göttliche Kunst (2019), pp. 80-93.

Herz, Josef (ed.): Das Tagebuch des Augsburger Arztes und Stadtphysicus Dr. Philipp Hoechstetter 1579-1635. In: Zeitschrift des Historischen Vereins für Schwaben und Neuburg 70 (1976), pp. 180-224.

Herzog, E.: Zwei alte Physikat-Bestallungen aus den Jahren 1523 und 1546. In: Vereinte deutsche Zeitschrift für die Staats-Arzneikunde, N.F. 3 (1848), nº 1, pp. 194-200.

Hess, Volker and Sabine Schlegelmilch: Cornucopia officinae medicae: Medical practice records and their origin. In: Dinges et alii, Medical practice (2016), pp. 11-38.

Hessus, Helius Eobanus: Helii Eobani Hessi [. . . ] et amicorum ipsius epistolarum familiarium libri XII. Marburg 1543.

Heusinger, Tobias: Das zitternde Herz des Monarchen. Kommentierte Edition eines ärztlichen Konsils von Andrea Gallo für Kaiser Maximilian II. Diss. med. Würzburg [2021] (in preparation).

Hild, Heike: Das Stammbuch des Medicus, Alchemisten und Poeten Daniel Stolcius als Manuskript des Emblembuches Viridarium Chymicum (1624) und als Zeugnis seiner Peregrinatio Academica. Diss. TU Munich 1991.

Hindson, Betham: Attitudes towards menstruation and menstrual blood in Elizabethan England. In: Journal of social history 43 (2009), pp. 89-114. 
Hippocrates: Aphorismi cum Galeni commentariis. Transl. by Niccolò Leoniceno. Venice 1538. Hippocrates: Euvres complètes d'Hippocrate. Ed. by Émile Littré. Paris 1839-1861 (repr. Amsterdam 1978).

Hippocrates: De aere, aquis et locis libellus. Transl. by Janus Cornarius. Basel 1529.

Hirai, Hiro: Medical humanism and natural philosophy: renaissance debates on matter, life and the soul. Boston/Leiden 2011.

Hirai, Hiro: The new astral medicine. In: Brendan Dooley (ed.): A companion to astrology in the Renaissance. Leiden/Boston 2014, S. 267-286.

Hirn, Josef: Erzherzog Ferdinand II. von Tirol. Geschichte seiner Regierung und seiner Länder. 2 vols. Innsbruck 1885/1887.

Hlaváčková, Ludmila and Petr Svobodný: Dějiny lékařství v českých zemích. Prague 2004.

Hlaváčková, Ludmila, Petr Svobodný and Josef Adamec: Biografický slovník pražské lékařské fakulty 1348-1939. 2 vols. Prague 1988 and 1993.

Hoffmann, Friedrich: Medicus politicus sive regulae prudentiae secundum quas medicus juvenis studia sua \& vitae rationem dirigere debet, si famam sibi felicemque praxin \& cito acquirere \& conservare cupit. Leiden 1708.

Hoffmann-Axthelm, Walter: Die Geschichte der Zahnheilkunde. Berlin 1973.

Hoppe, Brigitte: Bildungseifrige Apotheker der Frühen Neuzeit. In: Pharmazeutische Zeitung 137 (1992), n. 44, pp. 38-44.

Horer, Ananius: Artzney-Teuffel, oder kurtzer Discurs, darinn diesem Ertzmörder seine Larve abgezogen. Sine loco 1634.

Hörnigk, Ludwig von: Politia medica. Frankfurt am Main 1636.

Hornung, Johannes: De uroscopia fraudulenta discursus. Kurtzer Bericht von dem unvollkommenen und betrüglichen Urtheil des menschlichen Borns oder Harns. Herborn 1611.

Hornung, Johannes (ed.): Cista medica. Nürnberg [1626].

Horský, Zdeněk: Die europäische Bedeutung der böhmischen Tradition der "neuen

Wissenschaft" im 16. Jahrhundert. In: Hans-Bernd Harder (ed.): Studien zum Humanismus in den böhmischen Ländern. Cologne/Vienna 1988, pp.275-289.

Horst, Gregor: Büchlein von dem Schorbock, gemynem Vatterlandt zum besten Teutsch beschrieben. Gießen 1615.

Horst, Horst: Observationum medicinalium singularium libri quatuor posteriores. Ulm 1628.

Horst, Jakob: Brevis et dilucida enarratio libri Hippocratis De corde (with other texts connected to Horst's doctoral exam). Frankfurt an der Oder 1563.

Horst, Jakob: Oratio de remoris discentium medicinam earumque remediis. In: idem: Epistolae philosophicae et medicinales. Leipzig 1596, pp. 530-593.

Houdt, Toon van et alii (eds): Self-presentation and social identification. The rhetoric and pragmatics of letter-writing in early modern times. Leuven 2002.

Houllier, Jacques: De morborum internorum curatione liber I. Paris 1567.

Huber, Katharina: Felix Platters “Observationes”. Studien zum frühneuzeitlichen Gesundheitswesen in Basel. Basel 2003.

Hubmann, Astrid: Der Zahnwurm. Die Geschichte eines volksheilkundlichen Glaubens. Diss. med. Univ. Regensburg 2008.

Hutten, Ulrich von: Von der wunderbarlichen Artzney des Holtz Guaiacum genant, und wie man die Frantzosen oder Blatteren heilen sol. Transl. by Thomas Murner. Strasbourg 1519.

Ingegno, Alberto: Astrologia, magia e ordine del mondo. In: Pietro Rossi and Carlo A. Viano (eds): Storia della filosofia. 3. Dal Quattrocento al Seicento. Roma/Bari 1995, pp. 85-113. 
Ijsewein, Jozef: Companion to Neo-Latin studies. Part 2: Literary, linguistic, philological and editorial questions. $2^{\text {nd }}$ edn. Leuven 1998.

Institoris, Heinrich: Malleus maleficarum. Cologne 1511.

Jackson, Mark: Asthma. The biography. New York 2009.

Jackson, Stanley W.: Melancholia and depression. From Hippocratic times to modern times. New Haven/London 1986.

Jacquart, Danielle: Theory, everyday practice, and three fifteenth-century physicians. In: Osiris 6 (1990), pp. 140-160.

Jakubcová, Alena, Matthias Johannes Pernerstorfer and Hubert Reitterer: Theater in Böhmen, Mähren und Schlesien. Von den Anfängen bis zum Ausgang des

18. Jahrhunderts. Ein Lexikon. Vienna 2013.

Jaumann, Herbert: latrophilologia. "Medicus philologicus" und analoge Konzepte in der frühen Neuzeit. In: Ralph Häfner (ed.): Philologie und Erkenntnis. Beiträge zu Begriff und Problem frühneuzeitlicher "Philologie". Tübingen 2001, pp.151-176.

Jenny, Beat Rudolf (ed.): Die Amerbachkorrespondenz. Vol. 6 and Vol. 9,1. Basel 1967 and 1982.

Jensen, Kristian: The humanist reform of Latin and Latin teaching. In: Jill Kraye (ed.): The Cambridge companion to Renaissance humanism. Cambridge/New York 1996, pp. 63-81. Jewson, Nicholas D.: Medical knowledge and the patronage system in $18^{\text {th }}$ century England. In: Sociology 8 (1974), pp. 369-385.

Jewson, Nicholas D.: The disappearance of the sick-man from medical cosmology, 1770-1870.

In: Sociology 10 (1976), 225-244.

Jones, Colin: The smile revolution in eighteenth century Paris. Oxford 2014

Jouanna, Jacques: Die Entstehung der Heilkunst im Westen. In: Mirko Dražen Grmek (ed.): Die Geschichte des medizinischen Denkens. Antike und Mittelalter. Munich 1996, pp.28-80. Jouanna, Jacques: Hippocrates. In: Brunschwig/Lloyd, Greek thought (2000), pp. 649-659. Joubert, Laurent: Le première et seconde parti des erreurs populaires, touchant la médecine et le regime de santé. Rouen 1601.

Joubert, Laurent: Oratio de praesidiis futuri excellentis medici. Geneva 1580.

Joutsivuo, Timo: Scholastic tradition and humanist innovation. The concept of neutrum in Renaissance medicine. Helsinki 1999.

Junghans, Helmar: Zeitpunkt und Ort von Luthers Turmerlebnis angesichts neuer

Ausgrabungen. In: Christopher Spehr (ed.): Lutherjahrbuch 84 (2017), pp. 11-50.

Jütte, Robert: Ärzte, Heiler und Patienten. Medizinischer Alltag in der frühen Neuzeit. Munich/ Zürich 1991.

Jütte, Robert: “Wo kein Weib ist, da seufzet der Kranke”. Familie und Krankheit in der Frühen Neuzeit. In: Jahrbuch des Instituts für Geschichte der Medizin der Robert Bosch Stiftung 7 (1989), pp. 7-24.

Kalina von Jätenstein, Matthias: Nachrichten über böhmische Schriftsteller und Gelehrte. 2 vols. Prague 1818/1819.

Karcher, Johannes: Thomas Erastus (1524-1583), der unversöhnliche Gegner des

Theophrastus Paracelsus. In: Gesnerus 14 (1957), pp. 1-13.

Kaartinen, Marjo: “Pray, Dr, is there reason to fear a cancer?' Fear of breast cancer in early modern Britain.” In: Jonas Liliequist (ed.): A history of emotions, 1200-1800. London 2012, pp. 153-166 and pp. 241-243 (notes).

Kassell, Lauren: Casebooks in early modern England. Medicine, astrology, and written records. In: Bulletin of the history of medicine 88 (2014), pp. 595-625. 
Kassell, Lauren: Medicine and magic in Elizabethan London: Simon Forman - astrologer, alchemist, and physician. Oxford 2005.

Kegler, Caspar: [Quaestiones de vacuationibus purgationibusque medicinae studiosis disputandae]. Broadsheet. [Leipzig] c. 1500.

Kerger, Martin: Methodus excerpendi, Drexeliana succinctior (= appended to Drexel, Aurifodina). Breslau 1695.

Kijper, Albert: Medicinam rite discendi et exercendi methodus. Leiden 1643.

King, Helen: Hippocrates' woman. Reading the female body in ancient Greece. London/ New York 1998.

King, Helen: Midwifery, obstetrics and the rise of gynaecology. The uses of a sixteenth-century compendium. Aldershot 2007.

King, Helen: Once upon a text. Hysteria from Hippocrates. In: Sander I. Gilman et alii (eds): Hysteria beyond Freud. Berkeley 1993, pp. 3-90.

King, Helen: The one-sex body on trial. The classical and early modern evidence. Farnham 2013.

Kintzinger, Martin: Status medicorum. Mediziner in der städtischen Gesellschaft des 14. bis 16. Jahrhunderts. In: Peter Johanek (ed.): Städtisches Gesundheits- und Fürsorgewesen vor 1800. Cologne 2000, pp. 63-92.

Kinzelbach, Annemarie: Gesundbleiben, Krankwerden, Armsein in der frühneuzeitlichen Gesellschaft 1500 -1700. Gesunde und Kranke in den Reichsstädten Ulm und Überlingen. Stuttgart 1995.

Kinzelbach, Annemarie: Heilkundige Frauen im oberdeutschen Raum, 1450-1700. In: Historische Anthropologie 7 (1999), pp. 165-190.

Kinzelbach, Annemarie: Zur Sozial- und Alltagsgeschichte eines Handwerks in der frühen Neuzeit. "Wundärzte” und ihre Patienten in Ulm. In: Ulm und Oberschwaben 49 (1994), pp. 111-144.

Kinzelbach, Annemarie, Stephanie Neuner und Karen Nolte: Medicine in practice. Knowledge, diagnosis and therapy. In: Dinges et alii, Medical practice (2016), pp. 99-130.

Kirwan, Richard: Introduction: Scholarly self-fashioning and the cultural history of universities. In: idem (ed.): Scholarly self-fashioning and community in the early modern university. Farnham 2013, pp. 1-20.

Kistner, Nikolaus: Opuscula historica et politico-philologa tributa in libros IV. Ed. by Quirin Reuter. Frankfurt 1611.

Kitti, Jurina: Vom Quacksalber zum Doctor medicinae. Die Heilkunde in der deutschen Graphik des 16. Jahrhunderts. Cologne 1985.

Klaas, Philip, Hubert Steinke and Alois Unterkircher: Daily business. The organization and finances of doctors' practices. In: Dinges et alii, Medical practice (2016), pp. 71-98.

Klestinec, Cynthia: Theaters of anatomy. Students, teachers, and traditions of dissection in Renaissance Venice. Baltimore 2011.

Klibansky, Raymond, Erwin Panofsky and Fritz Saxl: Saturn und Melancholie. Studien zur Geschichte der Naturphilosophie und Medizin, der Religion und der Kunst. Frankfurt 1992.

Klose, Wolfgang: Corpus alborum amicorum. Beschreibendes Verzeichnis der Stammbücher des 16. Jahrhunderts. Stuttgart 1988.

Knoedler, Franz (ed.): De egestionibus: Texte und Untersuchungen zur spätmittelalterlichen Koproskopie. Pattensen 1979. 
Koch, Hans Theodor: Anatomie als universitäres Lehrfach. In: Jürgen Helm and Karin Stukenbrock (eds): Anatomie. Sektionen einer medizinischen Wissenschaft im 18. Jahrhundert. Wiesbaden 2003, pp. 163-188.

Koch, Hans Theodor: Die Wittenberger Medizinische Fakultät (1502-1652). Ein biobibliographischer Überblick. In: Stefan Oehmig (ed.): Medizin und Sozialwesen in Mitteldeutschland zur Reformationszeit. Leipzig 2007, pp. 289-343.

König, Klaus G.: Der Nürnberger Stadtarzt Dr. Georg Palma (1543-1591). Stuttgart 1961. Koning, Jan de: Lo sviluppo della botanica nel XVI secolo. In: Minelli, L'orto (1995), pp. 11-31. Kostenzer, Otto: Die Leibärzte Kaiser Maximilians I. in Innsbruck. In: Veröffentlichungen des Tiroler Landesmuseums Ferdinandeum 50 (1970), pp. 73-111.

Kotthorst, Lotte: Gelehrte Mediziner am Niederrhein. Das Italienstudium der Ärzte am Hof Wilhelms V. von Jülich-Kleve-Berg (1539-1592). In: Kaspar Gubler and Rainer C. Schwinges (eds): Gelehrte Lebenswelten im 15. und 16. Jahrhundert. Zürich 2018, pp. 129-156.

Kraack, Gerhard: Die Anfänge der medizinischen Versorgung in Flensburg und die Gründung der Ratsapotheke im Jahr 1604. In: Broder Schwensen (ed.): Flensburg um 1600. Flensburg 2006, pp. 283-306

Kragius, Andreas: Laurea apollinea monspelliensis. Basel 1586.

Kramarczyk, Andrea: Der Arzt Johannes Naevius (1499-1574) - ein Freund des Joachim Camerarius. In: Rainer Kößling and Günther Wartenberg (eds): Joachim Camerarius. Tübingen 2017, pp. 337-348.

Kramarczyk, Andrea and Antonia Krüger (eds): Im Dienste von Kaiser und Kurfürst. Die Leibärzte Johannes und Caspar Neefe und ihre Familie. Chemnitz 2014.

Kühlmann, Wilhelm: Poet, Chymicus, Mathematicus. Das Stammbuch des böhmischen Paracelsisten Daniel Stoltzius. In: Joachim Telle (ed.): Parerga Paracelsica. Paracelsus in Vergangenheit und Gegenwart. Stuttgart 1991, pp. 275-300.

Kühlmann, Wilhelm and Joachim Telle (eds): Der Frühparacelsismus. Vol. 1. Tübingen 2001.

Kühlmann, Wilhelm and Joachim Telle: Humanismus und Medizin an der Universität Heidelberg im 16. Jahrhundert. In: Wilhelm Doerr (ed.): Semper apertus. Sechshundert Jahre Ruprecht-Karls-Universität Heidelberg 1386-1986. Vol. 1: Mittelalter und Frühe Neuzeit 1386-1803. Berlin 1985, pp. 255-289.

Kühnel, Harry: Pietro Andrea Matthioli. Leibarzt und Botaniker des 16. Jahrhunderts. In: Mitteilungen des Österreichischen Staatsarchivs 15 (1962), pp. 63-92.

Kuhn, Werner: Die Studenten der Universität Tübingen zwischen 1477 und 1534. Ihr Studium und ihre spätere Lebensstellung. Göppingen 1971.

Kusukawa, Sachiko: Aspectio divinorum operum: Melanchthon and astrology for Lutheran medics. In: Ole Peter Grell and Andrew Cunningham (eds): Medicine and the Reformation. London 1993, pp. 33-56.

Kutschmann, Werner: Der Naturwissenschaftler und sein Körper. Die Rolle der "inneren Natur" in der experimentellen Naturwissenschaft der frühen Neuzeit. Frankfurt am Main 1986.

Kutschmann, Werner: Der Naturwissenschaftler und sein Körper. Naturwissensschaftsgeschichte aus antrhopologischer Perspektive. In: Berichte zur Wissenschaftsgeschichte 14 (1991), pp. 137-146.

Kutzer, Michael: Herrgott, Heiler und Harnschau: Das Vermächtnis des Ulmer Stadtarztes Augustin Thoner (1567-1655). In: Medizinhistorisches Journal 35 (2000), pp. 149-173. Labouvie, Eva: Andere Umstände. Eine Kulturgeschichte der Geburt. Cologne 1998. Labouvie, Eva: Verbotene Künste. Volksmagie und ländlicher Aberglaube in den Dorfgemeinden des Saarraumes (16.-19. Jahrhundert). St. Ingbert 1992. 
Lachmund, Jens and Gunnar Stollberg: Patientenwelten. Krankheit und Medizin vom späten 18. bis zum frühen 20. Jahrhundert im Spiegel von Autobiographien. Opladen 1995.

Lachmund, Jens and Gunnar Stollberg: The doctor, his audience, and the meaning of illness. The drama of medical practice in the late $18^{\text {th }}$ and early $19^{\text {th }}$ centuries. In: iidem (eds): The social construction of illness. Illness and medical knowledge in past and present. Stuttgart 1992, pp. 53-66.

Lahr, Beer: Original-Denkwürdigkeiten eines Zeitgenossen am Hofe Johann Wilhelm's III. Herzogs von Jülich, Cleve, Berg. Nebst einem Anhange von Original-Briefen und Verhandlungen betreffend den Proceß der Herzogin Jakobe. Ed. by E[rich] K[ühlwetter] und F[ranz Wilhelm] C[ustodis]. Düsseldorf 1834.

Laín Entralgo, Pedro: La historica clinica. Historia y teoria del relato patografico. Barcelona 1961. Laín Entralgo, Pedro: La relacion medico-enfermo. Historia y teoria. Madrid 1964.

Lambeck, Peter: Commentariorum de Augustissima Bibliotheca Caesarea Vindobonensi liber. Ed. by Adam Frantisek Kollár. $2^{\text {nd }}$ edn. Vienna 1769.

Lammel, Hans-Uwe: Hofmedizin als interdisziplinäre Forschungsaufgabe - eine Bilanz. In: Medizinhistorisches Journal 53 (2018), pp. 197-216.

Lane Furdell, Elizabeth: The royal doctors 1485-1714. Medical personnel at the Tudor and Stuart courts. New York 2001.

Lange, Johannes: Epistolarum medicinalium volumen tripartitum. Frankfurt 1589.

Lange, Johannes: Medicinalium epistolarum miscellanea, varia ac rara. Basel 1554.

Lange, Johannes: Secunda medicinalium epistolarum miscellanea, varia ac rara. Basel 1560.

Laqueur, Thomas W.: Making sex. Body and gender from the Greeks to Freud. Cambridge, MA/ London 1990.

Laschinger, Johannes: Dr. Hartmann Schedel als Stadtarzt in Amberg (1477-1481). In: Mitteilungen des Vereins für Geschichte der Stadt Nürnberg 80 (1993), pp. 137-145.

Lawrence, Christopher (ed.): Medical theory, surgical practice. Studies in the history of surgery. London/New York 1992.

Lawrence, Christopher: Democratic, divine and heroic. The history and historiography of surgery. In: Lawrence, Medical theory (1992), pp. 1-47.

Lawrence, Christopher: Medical minds, surgical bodies. corporeality and the doctors. In: Lawrence/Shapin, Science incarnate (1998), pp. 156-201.

Lawrence, Christopher and Steven Shapin (eds): Science incarnate. Historical embodiments of natural knowledge. Chicago 1998.

Ledvinka, Václav and Jiři Pešek: The public and private lives of Prague’s burghers. In: Fučíková, Rudolf II (1997), pp. 287-301.

Leinkauf, Thomas: Die Philosophie des Humanismus und der Renaissance. Munich 2020.

Leong, Elaine: Collecting knowledge for the family: Recipes, gender and practical knowledge in the early modern English household. In: Centaurus 55 (2013), pp. 81-103.

Leong, Elaine: Recipes and everyday knowledge. Medicine, science and the household in early modern England. Chicago/London 2018.

Lesser, Andreas: Die albertinischen Leibärzte vor 1700 und ihre verwandtschaftlichen Beziehungen zu Ärzten und Apothekern. Petersberg 2015.

Leven, Karl-Heinz: Die Geschichte der Infektionskrankheiten. Von der Antike bis ins 20. Jahrhundert. Landsberg 1997.

Leven, Karl-Heinz: Krankheiten - historische Deutung versus retrospektive Diagnose. In: Norbert Paul and Thomas Schlich (eds): Medizingeschichte. Aufgaben, Probleme, Perspektiven. Frankfurt 1998, pp. 153-185. 
Liber decanorum fac[ultatis] phil[sophiae] ab anno 1367, usque ad annum 1585. Part 2. Prague 1832.

Liébault, Jean: Trois livres appartenans aux infirmitez et maladies des femmes. Paris 1582. Lind, L. R.: Pre-Vesalian anatomy. Biography, translations, documents. Philadelphia 1975. Lindeboom, G. A.: Medical education in the Netherlands 1575-1750. In: C. D. O'Malley (ed.): The history of medical education. Berkeley 1970, pp. 201-234.

Lindemann, Mary: Medicine and society in early modern Europe. $2^{\text {nd }}$ edn. Cambridge 2010.

Lingo, Alison Klairmont: The rise of medical practitioners in sixteenth-century France. The case of Lyon and Montpellier. PhD-Dissertation. Berkeley 1980.

Lingo, Alison Klairmont: Empirics and charlatans in early modern France. In: Journal of social history 19 (1986), pp. 583-603.

Liphimeus, Sabalathrus: Warnung wider den Harn-Teuffel. Nürnberg 1626.

Lockwood, D. P.: Ugo Benzi. Medical philosopher and physician, 1376-1439. Chicago 1951.

Long, Pamela O.: Artisan/practitioners and the rise of the new science, 1400-1600. Corvallis, OR 2001.

Lonie, lain M.: Fever pathology in the sixteenth century. Tradition and innovation. In: William F. Bynum and Vivian Nutton (eds): Theories of fever from antiquity to the Enlightenment. London 1981, pp. 19-44.

Lonie, lain M.: The "Paris Hippocratics". Teaching and research in Paris in the second half of the sixteenth century. In: Wear, French and Lonie, Medical Renaissance (1985), pp. 169-174.

López Pinero, José María: Ciencia y técnica en la sociedad espanola de los siglos XVI y XVII. Barcelona 1979.

López Pinero, José María: The medical profession in $16^{\text {th }}$ century Spain. In: Russell, Town and state physician (1981), pp. 85-98.

Löwenstein, Jakob Samuel: Biographien und Schriften der ordentlichen Professoren der Medicin an der Hochschule zu Frankfurth a.O. in den Jahren 1506 bis 1811. In: Janus (1848), pp. 283-315 and pp. 419-443.

Low, Setha M: Culturally interpreted symptoms or culture-bound syndromes. A cross-cultural review of nerves. In: Social science and medicine 21 (1985), pp. 187-196.

Low, Setha M: Embodied metaphors: nerves as lived experience. In: Thomas J. Csordas (ed.): Embodiment and experience. The existential ground of culture and self. Cambridge 1994, pp. 139-162.

Ludovicus, Laurentius (ed.): Compendium etymologiae et syntaxis in usum gymnasii Gorlicensis. Addita sunt gnorismata regularum in syntaxi, usurpata a Valentino Trozedorfio, in schola Goldbergensi. Görlitz 1572.

Ludwig, Walther: Das Stammbuch als Bestandteil humanistischer Kultur. Das Album des Heinrich Carlhack Hermeling (1587-1592). Göttingen 2006.

Ludwig, Walther (ed.): Vater und Sohn im 16. Jahrhundert. Der Briefwechsel des Wolfgang Reichart genannt Rychardus mit seinem Sohn Zeno (1520-1543). Hildesheim 1999.

Lunel, Alexandre: La maison médicale du roi. XVIe-XVIIle siècle. Le pouvoir royal et les professions de santé. Seyssel 2008.

Luther, Martin: Werke. Kritische Gesamtausgabe. Briefwechsel. Vol. 5: 1529-1530. Ed. by O. Clemen. Weimar 1934.

MacDonald, Michael: Mystical Bedlam. Madness, anxiety, and healing in seventeenth-century England. Cambridge 1981. 
Mache, Ursula: Anatomischer Unterricht in Padua im 16. Jahrhundert. Edition, Übersetzung und Kommentierung der Aufzeichnungen eines böhmischen Studenten. Duisburg 2019 (= Diss. med. dent. Regensburg 2019).

MacKinney, Loren C.: Medical education in the Middle Ages. In: Cahiers d'histoire mondiale 2 (1955), pp. 835-861.

MacKinney, Loren C.: Medical ethics and etiquette in the early middle ages. The persistence of Hippocratic ideals. In: Bulletin of the history of medicine 26 (1952), pp. 1-31.

Maclean, Ian: Logic, signs and nature in the Renaissance. The case of learned medicine. Cambridge 2002.

Maclean, Ian: The medical republic of letters before the Thirty Years War. In: Intellectual history review 18 (2008), pp. 15-30.

Maclean, Ian: The Renaissance notion of woman. A study in the fortunes of scholasticism and medical science in European intellectual life. Cambridge 1987.

Maiwald, V[incenz]: Geschichte der Botanik in Böhmen. Vienna/Leipzig 1904.

Malatesta, Maria (ed.): Doctors and patients. History, representation, communication from antiquity to the present. Berkeley 2015.

Manardi, Giovanni: Epistolae medicinales, in quibus multa recentiorum errata et antiquorum decreta reserantur. Ferrara 1521.

Manardi, Giovanni: Epistolarum medicinalium libri XX. Venice 1557.

Mantese, Giovanni: Per una storia dell'arte medica in Vicenza alla fine del sec. XVI. Vicenza 1969.

Manzke, Walter: Remedia pro infantibus. Arzneiliche Kindertherapie im 15. und 16. Jahrhundert, dargestellt anhand ausgewählter Krankheiten. Diss. rer. nat. Marburg 2008.

Marinello, Giovanni: Le medicine partenenti alle infermità delle donne. Venice 1563.

Marland, Hilary: Dangerous motherhood. Insanity and childbirth in Victorian Britain. Basingstoke/New York 2004.

Martínek, Jan: Jan Hodějovský a jeho literární okruh. Ed. by Marta Vaculínová, together with Dana Martínková. Prague 2012.

Martínková, Dana: Beschreibungen böhmischer und mährischer Städte im Zeitalter des Humanismus. In: Hans-Bernd Harder (ed.): Studien zum Humanismus in den böhmischen Ländern. Part III: Die Bedeutung der humanistischen Topographien und Reisebeschreibungen in der Kultur der böhmischen Länder bis zur Zeit Balbins. Cologne 1993, pp.25-34.

Martínková, Dana: Literárni druh veršovaných popisů měst v naši latinské humanistické literatuře. Posthumous edition by Marta Vaculínová (together with Martínek, Jan Hodějovský). Prague 2012.

Martínková, Dana: Poselství ducha. Latinská próza českých humanistů. Prague 1975.

Marzell, Heinrich: Die Haselwurz (Asarum europaeum L.) in der alten Medizin. In: Sudhoffs Archiv für Geschichte der Medizin und der Naturwissenschaften 42 (1958), pp. 319-325.

Massaria, Alessandro: Praelectiones de morbis mulierum. Ed. by Heinrich Osthausen. Leipzig 1600.

Mattioli, Pietro Andrea: Commentarii in libros sex Pedacii Dioscoridis de materia medica. Venice 1554.

Mattioli, Pietro Andrea: Commentarii in P. Dioscoridis De materia medica. Venice 1565.

Mattioli, Pietro Andrea: Commentarii in sex libros Pedacii Dioscoridis Anazarbei de medica materia. Venice 1570.

Mattioli, Pietro Andrea: Epistolarum medicinalium libri quinque. Prague 1561.

Mattioli, Pietro Andrea: Epistolarum medicinalium libri quinque. Lyon 1564. 
Mattioli, Pietro Andrea: Il Magno Palazzo del Cardinale di Trento. Venice 1539.

Mattioli, Pietro Andrea: Morbi gallici novum ac utilissimum opusculum. In: Niccolò Leoniceno et alii: Liber de morbo gallico. Venice 1535 [no pagination].

Mattioli, Pietro Andrea: New Kreutterbuch mit den allerschönsten und artlichsten Figuren aller Gewechsz, dergleichen vormals in keiner Sprach nie an Tag kommen. Transl. and ed. by Georg Handsch. Venice 1563.

Mattioli, Pietro Andrea: Kreutterbuch. Ed. by Joachim Camerarius. Frankfurt am Main 1586.

Mauch, Adolf: Libellus de aegritudinibus infantium. Ein Buch über Kinderkrankheiten von Paolo Bagellardi (Padua 1472), ins Deutsche übertragen. Diss. med. Bottrop 1937.

Mauss, Marcel: Die Gabe. Form und Funktion des Austauschs in archaischen Gesellschaften. Frankfurt 1990.

Mayer, Maximilian: Verständnis und Darstellung des Skorbuts im 17. Jahrhundert. Mit einer Edition und Übersetzung der Fallgeschichten zu 'Skorbut' bei Johannes Frank. Diss. med. Würzburg 2012 (http://opus.bibliothek.uni-wuerzburg.de/frontdoor/index/index/docld/6241).

Mazzetti, Serafino: Repertorio di tutti i professori antichi, e moderni della famosa Università, $e$ del celebre Istituto delle Scienze di Bologna. Bologna 1847.

Mazzuchelli, Giammaria: Gli scrittori d'Italia cioè notizie storiche, e critiche intorno alle vite, $e$ agli scritti dei letterati Italiani. Vol. 1.1. Brescia 1758.

McClive, Cathy: Menstruation and procreation in early modern France. Farnham 2015.

McVaugh, Michael R.: The rational surgery of the Middle Ages. Tavarnuzze/Impruneta 2006.

Meibom, Johann Heinrich: Hippocratis magni Orkos [graece] sive jusjurandum, recensitum, et libro commentario illustratum. Leiden 1643.

Melanchthon, Philipp: Commentarius de anima. Wittenberg 1540.

Melanchthon, Philipp: Liber de anima. Wittenberg 1552.

Melanchthon, Philipp: Loci communes rerum theologicarum seu hypotyposes theologicae. Wittenberg 1521.

Melhofer, Philipp: Lasstafel oder Almannach [. . . .] Auff das MDXLIII Jare. Augsburg 1543.

Menčik, Ferdinand (ed.): Dopisy M. Matouše Kollína z Chotěřiny a jeho přátel ke Kašparovi z Nydbrucka, tajnému radovi krále Maximiliána II. Prague 1914.

Menini, Cesare: “Curationes A. M. Brasavoli”. Contributo alla conoscenza delle opere di Antonio Musa Brasavolo come medico pratico. In: Rivista della storia delle scienze mediche e naturali 43 (1952), pp. 255-261.

Mercado, Luìs: De mulierum affectionibus. Venice 1587.

Mercuriale, Girolamo: De morbis cutaneis. Transl. by Richard L. Sutton as: Sixteenth century physician and his methods. Mercurialis on diseases of the skin. Kansas City 1986.

Mercuriale, Girolamo: De morbis muliebribus praelectiones. $4^{\text {th }}$ edn. Venice 1601.

Mercuriale, Girolamo: De morbis puerorum tractatus. Venice 1583.

Mercuriale, Girolamo: De ratione discendi medicinam epigraphe. In: Joachim Georg Schenck, (ed.): De formandis medicinae studiis et schola medica constituenda enchiridion selectum. Strasbourg 1607, pp. 18-35.

Merenda, Giovanni P.: Evacuandi ratio tribus in libris luculenter perstricta. Basel 1547.

Mertens, Dieter: Zur Sozialgeschichte und Funktion des poeta laureatus im Zeitalter Maximilians I. In: Rainer Christoph Schwinges (ed.): Gelehrte im Reich. Zur Sozial- und Wirkungsgeschichte akademischer Eliten des 14. bis 16. Jahrhunderts. Berlin 1996, pp. 327-348.

Merula, Gaudenzio: Memorabilium opus. Lyon 1556.

Metzger, Nadine: Wolfsmenschen und nächtliche Heimsuchungen: Zur kulturhistorischen Verortung vormoderner Konzepte von Lykanthropie und Ephialtes. Remscheid 2011. 
Meyer, Stefanie: Der “Discursus medicus et politicus" von Tobias Geiger (1656). Edition und Kommentar. Diss. med. Würzburg 2021.

Micale, Mark S.: Approaching hysteria. Disease and its interpretations. Princeton 1995.

Mikkeli, Heikki: An Aristotelian response to Renaissance humanism. Jacopo Zabarella on the nature of arts and sciences. Helsinki 1992.

Mikkeli, Heikki: Hygiene in the early modern medical tradition. Helsinki 1999.

Miller, Genevieve: A seventeenth-century astrological diagnosis. In: Edgar Ashworth Underwood (ed.): Science, medicine and history. Essays on the evolution of scientific thought and medical practice. London 1953, pp. 27-33.

Milton, J. R.: Induction before Hume. In: British journal for the philosophy of science 38 (1987), pp. 49-74.

Moehsen, J. C. W.: Leben Leonhard Thurneissers zum Thurn. Ein Beitrag zur Geschichte der Alchemie wie auch der Wissenschaften und Künste in der Mark Brandenburg gegen Ende des 16. Jahrhunderts. Berlin/Leipzig 1783.

Minelli, Alessandro (ed.): L'orto botanico di Padova, 1545-1995. Venice 1998.

Mone, Franz Joseph: Ueber Krankenpflege, vom 13. bis 16. Jahrhundert. In: Zeitschrift für die Geschichte des Oberrheins 2 (1851), pp. 257-291.

Monnetus, Io[annis] Carolus: Ad lectorem. In: Giovanni Battista da Monte: In tertium primi Epidemiorum sectionem explanationes. Ed. by Valentinus Lublinus. Venice 1554.

Montagnana, Marco Antonio: De herpete, phagedaena, gangraena, sphacelo et cancro, tam cognoscendis, tam curandis tractatio accuratissima. Venice 1589.

Montaigne, Michel de: Die Essais. Selected and ed. by Arthur Franz. Leipzig 1953.

Moran, Bruce: The alchemical world of the German court. Occult philosophy and chemical medicine in the circle of Moritz of Hessen (1572-1632). Stuttgart 1991.

Moss, Ann: Power and persuasion. Commonplace culture in early modern Europe. In: David Cowling and Mette B. Bruun (eds): Commonplace culture in Western Europe in the early modern period. Leuven 2011, pp. 1-17.

Moss, Ann: Printed commonplace-books and the structuring of Renaissance thought. Oxford 1996.

Muccillo, Maria: Da Monte (De Monte, Dei Monte), Giovanni Battista. In: Dizionario biografico degli Italiani 32 (1986) (http://www.treccani.it/enciclopedia/da-monte-giovanni-battistadetto-montano_\%28Dizionario-Biografico\%29/).

Müller, Harald: “Specimen eruditionis". Zum Habitus der Renaissance-Humanisten und seiner sozialen Bedeutung. In: Frank Rexroth (ed.): Beiträge zur Kulturgeschichte der Gelehrten im späten Mittelalter. Ostfildern 2010, pp. 117-151.

Müller, Nikolaus: Philipp Melanchthons letzte Lebenstage, Heimgang und Bestattung nach den gleichzeitigen Berichten der Wittenberger Professoren. Zum 350. Todestage Melanchthons. Leipzig 1910.

Müller-Jahncke, Wolf-Dieter: Von Ficino zu Agrippa. Der Magia-Begriff des RenaissanceHumanismus im Überblick. In: Antoine Faivre and Rolf Christian Zimmermann (eds): Epochen der Naturmystik. Hermetische Traditionen im wissenschaftlichen Fortschritt. Berlin 1979, pp. 24-51.

Mugnai Carrara, Daniela: Epistemological problems in Giovanni Mainardi's commentary on Galen's Ars parva. In: Anthony Grafton and Nancy Siriasi (eds): Natural particulars. Nature and the disciplines in Renaissance Europe. Cambridge, MA 1999, pp. 251-273.

Mugnai Carrara, Daniela: Profilo di Nicolò Leoniceno. In: Interpres. Rivista di studi quattrocenteschi (1979), pp. 169-212. 
Mugnai Carrara, Daniela and Maria Conforti: L'insegnamento della medicina dall'istituzione delle università al 1550. In: Antonio Clericuzio and Germana Ernst (eds): /l Rinascimento italiano e l'Europa. Vol. 5: Le scienze. Treviso/Costabissara 2008, pp. 455-478.

Murphy, Hannah: A new order of medicine. The rise of physicians in Reformation Nuremberg. Pittsburgh 2019.

Nagy, Doreen Evenden: Popular medicine in seventeenth-century England. Bowling Green 1988.

Nance, Brian: Turquet de Mayerne as Baroque physician. The art medical portraiture. Amsterdam/New York 2001.

Neefe, Caspar: De missione sanguinis, quam phlebotomiam appellant, disputatio. Leipzig 1548.

Nelles, Paul: Reading and memory in the universal library: Conrad Gessner and the Renaissance book. In: Donald Beecher and Grant Williams (eds): Ars reminiscendi. Mind and memory in Renaissance culture. Toronto 2009, pp. 147-169.

Nettesheim, Agrippa von: Die Eitelkeit und Unsicherheit der Wissenschaft und die Verteidigungsschrift. Ed. by Fritz Mauthner. Vol. 2. Munich 1913.

Neuburger, Max: Die Lehre von der Heilkraft der Natur im Wandel der Zeiten. Stuttgart 1926.

Neumeister, Sebastian and Conrad Wiedemann (eds): Res publica litteraria. Die Institutionen der Gelehrsamkeit in der frühen Neuzeit. Wiesbaden 1987.

Neuser, Wilhelm H.: Das Stammbuch des Zacharias Ursinus (1553-1563 und 1581). In: Blätter für pfälzische Kirchengeschichte und religiöse Volkskunde 31 (1964), pp. 101-155.

Newton, Hannah: The sick child in early modern England, 1580-1720. Oxford 2012.

Nicoud, Marilyn: Medici, lettere e pazienti. Pratica medica e retorica nella corrispondenza della cancelleria sforzesca. In: Andretta/Nicoud, Être médecin (2013), pp. 213-233.

Nováková, Julie: Rytmické kalendarium Jiřího Handsche. In: Listy filologické 89 (1966), pp. 315-320.

Nutton, Vivian: Continuity or rediscovery? The city physician in classical antiquity and mediaeval Italy. In: Russell, Town and state physician (1981), pp. 9-46.

Nutton, Vivian.: Hippocrates in the Renaissance. In: Gerhard Baader and Rolf Winau (eds): Die Hippokratischen Epidemien. Theorie - Praxis - Tradition. Stuttgart 1989, pp. 420-439.

Nutton, Vivian: Humanist surgery. In: Wear, French and Lonie, Medical Renaissance (1985), pp. 75-99.

Nutton, Vivian: Introduction. In: idem, Medicine (1990), pp. 1-14.

Nutton, Vivian: John Caius und Johannes Lange. Medizinischer Humanismus zur Zeit Vesals. In: NTM 21 (1984), pp. 81-87.

Nutton, Vivian (ed.): Medicine at the courts of Europe, 1500-1837. London/New York 1989.

Nutton, Vivian: Medicine at the German Universities, 1348-1500. A Preliminary Sketch. In: Würzburger medizinhistorische Mitteilungen (1997), pp. 173-187.

Nutton, Vivian: Roman medicine, $250 \mathrm{BC}$ to $A D 200$, and medicine in late antiquity and the early Middle Ages. In: Conrad Lawrence et alii: The Western medical tradition, 800 BC to $A D$ 1800. Cambridge, 1995, pp. 39-70.

Nutton, Vivian: The diffusion of ancient medicine in the Renaissance. In: Medicina nei secoli N. S. 14 (2002), pp. 461-478.

Nutton, Vivian and Roy Porter (eds): The history of medical education in Britain. Amsterdam/ Atlanta,GA 1995.

Nutton, Vivian: The reception of Fracastoro's theory of contagion. The seed that fell among thorns? In: Osiris 6 (1990), pp. 196-234. 
Nutton, Vivian.: Wittenberg anatomy. In: Ole Peter Grell (ed.): Medicine and the Reformation. London 1993, pp. 11-32.

Oberrauch, Lukas: Medizin. In: Martin Korenjak (ed.): Tyrolis latina. Geschichte der lateinischen Literatur in Tirol. Vol. 1: Von den Anfängen bis zur Gründung der Universität Innsbruck. Vienna 2012, pp. 363-377.

Oetheus, Jakob: Gründtlicher Bericht, Lehr unnd Instruction von rechtem und nutzlichem Brauch der Artzney. Dillingen 1574.

Oetheus, Jakob: Theses de methodo therapeutica, secundum dogmaticam, ac rationalem medicinam. Resp. Philipp Menzel. Ingolstadt 1569.

Ofenhitzer, Franziska: Praxisalltag in der Frühen Neuzeit. Das Rezeptdiarium (1612-1616) von Petrus Kirstenius aus Breslau. Diss. med. Univ. Würzburg 2015.

Ogilvie, Brian W.: The science of describing. Natural history in Renaissance Europe. Chicago/ London 2006.

Olmi, Giuseppe: Molti amici in vari luoghi. Studio della natura e rapporti epistolari nel XVI secolo. In: Nuncius 6 (1991), pp. 3-31.

O’Malley, Charles D.: Andreas Vesalius of Brussels, 1514-1564. Berkeley/Los Angeles 1965.

O'Malley, Charles D.: Medical education during the Renaissance. In: idem (ed.): History of medical education. Berkeley/Los Angeles 1970, pp. 89-102.

Ongaro, Giuseppe: La medicina nello Studio di Padova e nel Veneto. In: Storia della cultura veneta. Dal primo Quattrocento al Consilio di Trento. Vol III/III. Vicenza 1981, pp. 75-134.

Ongaro, Giuseppe: L'insegnamento clinico di Giovan Battista da Monte (1489-1551). Una revisione critica. In: Physis 31 (1994), pp. 357-369.

Ongaro, Giuseppe: Medicina. In: Piero del Negro (ed.): L'Università di Padova. Otto secoli di storia. Padua 2001, pp. 153-193.

Ongaro, Giuseppe: Wirsung a Padova 1629-1643. Treviso 2010.

Orsolato, Giuseppe: Sulla prima fondazione di una clinica in Padova e sul monumento a G. B. Da Monte nella casa che fu del professore G. A. Giacomini. In: Rivista periodica dei lavori della Reale Accademia di scienze, lettere ed arti in Padova 23 (1872-73), pp. 127-152.

Padovani, Elideo: Processus, curationes et consilia in curandis in particularibus morbis quae prosperos habuerunt eventus. Ed. by Johannes Wittich. Leipzig 1607.

Pagel, Walter: Das medizinische Weltbild des Paracelsus. Seine Zusammenhänge mit Neuplatonismus und Gnosis. Wiesbaden 1962.

Palmer, Richard: Physicians and the state in post-medieval Italy. In: Russell, Town and state physician (1981), pp. 47-61.

Panáček, Jaroslav: Testament Georga Handsche z roku 1578. Bezděz 2013.

Pancino, Claudia: Doctor and patient in the modern age: words, gazes and gestures. In: Maria Malatesta (ed.): Doctors and patients. History, representation, communication from antiquity to the present. San Francisco 2015, pp. 81-107.

Pánek, Jaroslav: The nobility in the Czech lands, 1550-1650. In: Fučíková, Rudolf II (1997), pp. 270-286.

Panke-Kochinke, Birgit: Die Geschichte der Krankenpflege (1679-2000). Ein Quellenbuch. Frankfurt 2003.

Paracelsus: Grosse Wundartzney. Von allen Wunden, Stich, Schüsß Bränd, Bisß, Beynbrüch. Ulm 1536.

Paracelsus: Von der Bergsucht oder Bergkranckheiten drey Bücher. Dillingen 1567.

Paracelsus: Von der frantzösischen Kranckheit drey Bücher. Nürnberg 1530. 
Pardi, Giuseppe: Titoli dottorali conferiti dallo studio di Ferrara nei sec. XV e XVI. Lucca 1901 (repr. Bologna 1970).

Paré, Ambroise: Les œuvres. Paris 1575.

Paré, Ambroise: Opera chirurgica. Frankfurt 1594.

Paré, Ambroise: Response [. . . ] aux calomnies d'aucuns médecins, et chirurgiens, touchant ses œuvres. [Paris 1575].

Park, Katherine: Doctors and medicine in early Renaissance Florence. Princeton/New York 1985.

Park, Katharine: Observations in the margins, 500-1500. In: Lorraine Daston and Elizabeth Lunbeck (eds): Histories of scientific observation. Chicago/London 2011, pp. 15-44.

Park, Katharine and Robert A. Nye: Destiny is anatomy. In: The New Republic 18 February 1991, pp. 53-57.

Paullini, Christian Franz: Wie nemlich mit Koth und Urin fast alle, ja auch die schwerste, gifftige Kranckheiten, und bezauberte Schaden, vom Haupt biß zun Füssen inn- und äusserlich glücklich curirt worden. Frankfurt 1696.

Paulos von Aegina: Paulos' von Aegina, des besten Arztes sieben Bücher. Transl. and ed. by Julius Berendes. Leiden 1914.

Pawlik, Christian: Martin Stainpeis: Liber de modo studendi seu legendi in medicina. Bearbeitung und Erläuterung einer Studienanleitung für Mediziner im ausgehenden Mittelalter. Diss. med. TU Munich 1980.

Pellegrin, Pierre: Medicine. In: Brunschwig/Lloyd, Greek thought (2000), pp. 414-432.

Pennuto, Concetta: The debate on critical days in Renaissance Italy. In: Anna Akasoy, Charles Burnett and Ronit Yoeli-Tlalim (eds): Astro-medicine. Astrology and medicine, East an West. 2008, pp. 75-98.

Pešek, Jiří: Prague between 1550 and 1650. In: Fučíková, Rudolf II (1997), pp. 252-269.

Pfeil, Brigitte and Tilmann Walter: Im Dienst der Reichsstadt. Der spätmittelalterliche Stadtarzt Amplonius von der Buchen (1403-1438) und seine Briefe an die Stadt Nördlingen. In: Jahrbuch des Historischen Vereins für Nördlingen und das Ries 35 (2017), pp. 57-91.

Phaer, Thomas: The regiment of life [. . .] with the boke of children. London 1545.

Piccinini, Gabriella: Tra scienza ed arti. Lo Studio di Siena e l'insegnamento della medicina (secoli XIII-XVI). In: L'Università di Siena. 750 anni di storia. Milan 1991, pp. 145-158.

Pictorius, Georg: Von Zernichten Artzten. Strasbourg 1557.

Pieters, Jürgen and Julie Rogiest: Self-fashioning in de vroegmoderne literatuur- en cultuurgeschiedenis: genese en ontwikkeling van een concept. In: Frame 22 (2009), pp. 43-59.

Placotomus, Johannes [alias Johannes Brettschneider]: De ratione discendi ac praecipue medicinam. Leipzig 1552.

Planer, Andreas: Theses med[ico]-phys[icae] de concoctione, eiusque differentiis. Resp. Israel Spach. Strasbourg 1577.

Planerio, Giovanni: Epistolae morales. In: idem: Varia opuscula. Venice 1584 (separate pagination).

Planerio, Giovanni: Brevis patriae suae descriptio. In: idem: Varia opuscula. Venice 1584 (separate pagination).

Platter, Felix: Beschreibung der Stadt Basel 1610 und Pestbericht 1610/11. Ed. by Valentin Lötscher. Basel/Stuttgart 1987.

Platter, Felix: De corporis humani structura et usu libri III. Tabulis methodice explicati, iconibus accurate illustrati. Basel 1583. 
Platter, Felix: Observationum in hominis affectibus plerisque corpori et animo [. . .] incommodantibus libri tres. Basel 1614.

Platter, Felix: Quaestionum medicarum paradoxarum \& endoxarum, iuxta partes medicinae dispositarum centuria posthuma. Ed. by Thomas Platter. Basel 1625.

Platter, Felix: Tagebuch (Lebensbeschreibung) 1536-1567. Ed. by Valentin Lötscher. Basel 1976.

Plinius: Epistolarum libri X. Lyon 1539.

Pomata, Gianna: A word of the empirics: The ancient concept of observation and its recovery in early modern medicine. In: Annals of science 65 (2011), pp. 1-25.

Pomata, Gianna: La promessa di guarigione. Malati e curatori in antico regime. Bologna XVI-XVIII secolo. Bari 1994.

Pomata, Gianna: Observation rising. Birth of an epistemic genre, 1500-1600. In: Lorraine Daston and Elizabeth Lunbeck (eds): Histories of scientific observation. Chicago/London 2011, pp. 45-80.

Pomata, Gianna: Praxis historialis. The uses of historia in early modern medicine. In: Pomata/ Siraisi, Historia (2005), pp. 105-146.

Pomata, Gianna: Sharing cases: The observationes in early modern medicine. In: Early science and medicine 15 (2010), pp. 193-236.

Pomata, Gianna and Nancy G. Siraisi (eds): Historia. Empiricism and erudition in early modern Europe. Cambridge, MA 2005.

Pons, Jacobus: Medicus seu ratio, ac via aptissima ad recte tum discendam, tum exercendam medicinam. Ad tyrones. Lyon 1600.

Pormann, Peter E. and Emily Savage Smith: Medieval Islamic medicine. Cairo 2007.

Porter, Roy and George S. Rousseau: Gout. The patrician malady. New Haven/London 1998.

Porter, Roy: The rise of physical examination. In: W. F. Bynum and Roy Porter (eds): Medicine and the five senses. Cambridge 2004, pp. 179-197.

Poynter, F. N. L. and W. J. Bishop (eds): A seventeenth-century doctor and his patients: John Symcotts, 1592?-1662. Streatley 1951.

Premuda, Loris: Un discepolo di Leoniceno tra filologia ed empirismo. G. Manardo e il "libero esame" dei classici della medicina in funzione di più spreguidicati orientamenti metodologici. In: Atti del convegno internazionale per la celebrazione del V centenario della nascita di Giovanni Manardo 1462-1536. Ferrara 1963, pp. 43-56.

Prioreschi, Plinio: Did the Hippocratic physician treat hopeless cases? In: Gesnerus 49 (1992), pp. 341-350.

Procházka, Faustin: De saecularibus liberalium artium in Bohemia et Moravia fatis commentarius. Prague 1782.

Pulz, Waltraud: Nüchternes Kalkül - verzehrende Leidenschaft. Nahrungsabstinenz im 16. Jahrhundert. Cologne/Weimar/Vienna 2007.

Purš, Ivo: Die Bibliothek Erzherzog Ferdinands II. auf Schloss Ambras. In: Sabine Haag and Veronika Sandbichler (eds): Ferdinand II. 450 Jahre Tiroler Landesfürst. Innsbruck 2017, pp. 99-104.

Quaranta, Alessandra: Medici trentini e Respublica medicorum europea: scambi culturali e scientifici nella seconda metà del Cinquecento. In: Studi Trentini. Storia 97 (2018), pp. 83-120.

Questel, Caspar: Dissertatio academica de pulvinari morientibus non subtrahendo, von Abziehung der Sterbenden Haupt=Küssen, ex moralibus, divinis, juris item ac artis medicae principis methodice proposita, et exemplis rarioribus illustrata. Jena 1678. 
Ragland, Evan R.: "Making trials" in sixteenth- and early seventeenth-century European academic medicine. In: Isis 108 (2017), pp. 503-528.

Raimondi, C.: Lettere di P.A. Mattioli ad Ulisse Aldrovandi. In: Bullettino senese di Storia patria 13 (1906), pp. 121-185.

Ramsey, Matthew: Professional and popular medicine in France, 1770-1830. The social world of medical practice. Cambridge 1988.

Rankin, Alisha: Becoming an expert practitioner: Court experimentalism and the medical skills of Anna of Saxony (1532-1585). In: Isis 98 (2007), pp. 23-53.

Rankin, Alisha: On anecdote and antidotes. Poison trials in early modern Europe. In: Bulletin of the history of medicine 91 (2017), pp. 274-302.

Rankin, Alisha: Panaceia's daughters. Noblewomen as healers in early modern Germany. Chicago/London 2013.

Rankin, Alisha: The poison trials. Wonder drugs, experiment, and the battle for authority in Renaissance science. Chicago 2021.

Raphael, Lutz: Habitus und sozialer Sinn. Der Ansatz der Praxistheorie bei Pierre Bourdieu. In: Friedrich Jäger and Jürgen Straub (eds): Handbuch der Kulturwissenschaften. Vol. 2. Stuttgart 2004, pp. 266-276.

Rasori, Giovanni: Sul metodo degli studi medici prolusione. Milan 1809.

Rath, Gernot: Die Entwicklung des klinischen Unterrichts. Göttingen 1965.

Rath, Gernot: Moderne Diagnosen historischer Seuchen. In: Deutsche medizinische Wochenschrift 81 (1956), pp. 2065-2069.

Rather, L. J.: The genesis of cancer: A study in the history of ideas. Baltimore 1978.

Reckwitz, Andreas: Grundelemente einer Theorie sozialer Praktiken. Eine sozialtheoretische Perspektive. In: Zeitschrift für Soziologie 32 (2003), pp. 282-301.

Read, Sara: Menstruation and the female body in early modern England. Basingstoke 2013.

Reeds, Karen Meier: Botany in medieval and Renaissance universities. New York/London 1991.

Reger, Brigitte: Affectio hypochondriaca. Das Krankheitsbild der Hypochondrie in der Frühen Neuzeit. Diss. med. Regensburg 2015.

Resende, Garcia de: Crónica de D. João Il e miscelânea. Coimbra 1798 (facsimile reprint Lisbon 1991).

Renner, Franz: Ein new wolgegründet nützlichs unnd haylsams Handtbüchlein gemeiner Praktik aller innerlicher und eusserlicher Erzney wider die Krankheit der Franzosen. Nürnberg 1557.

Reusnerus, Nicolaus: Icones sive imagines virorum literis illustrium. Strasbourg 1590.

Rhazes (Al-Rāzī): Al-Rāzī, on the treatment of small children (De curis puerorum). The Latin and Hebrew translations. Ed. by Michael McVaugh. Leiden 2015.

Richards, Jennifer: Useful books: Reading vernacular regimens in sixteenth-century England. In: Journal of the history of ideas 73 (2012), pp. 247-271.

Richardson, Linda Deer: The generation of disease. Occult causes and diseases of the total substance. In: Wear, French und Lonie, Medical Renaissance (1985), pp. 175-194.

Richter, David: Genealogia Lutherorum. Berlin/Leipzig 1733.

Ridder-Symoens, Hilde de (ed.): A history of the university in Europe. Vol. II: Universities in early modern Europe (1500-1800). Cambridge 1996.

Riddle, J. M.: Three previously unknown sixteenth-century contributors to pharmacy, medicine and botany. Ioannes Manardus, Franciscus Frigimelica and Melchior Guilandinus. In: Pharmacy in history 21 (1979), pp. 143-155. 
Ritzmann, Iris: Sorgenkinder. Kranke und behinderte Mädchen und Jungen im 18. Jahrhundert.

Cologne/Weimar/Vienna 2008.

Roeck, Bernd: Der Morgen der Welt. Geschichte der Renaissance. Munich 2017.

Roger, Jacques: Jean Fernel et les problèmes de la médecine de la Renaissance. Paris 1960.

Rondelet, Guillaume: Libri de piscibus marinis in quibus verae piscium effigies expressae sunt. Lyon 1554.

Ronsseus, Balduinus: Miscellanea seu epistolae medicinales. Leiden 1590.

Rosenberg, Daniel: Early modern information overload. In: Journal of the history of ideas 64 (2003), pp. 1-9.

Rosenheim, Max: The album amicorum. Communicated to the Society of Antiquaries. Oxford 1910.

Rudel, Otto: Beiträge zur Geschichte der Medizin in Tirol. Gesammelt für das Etschländer Ärzteblatt. Bozen 1925.

Ründal, Erik O.: “daß seine Mannschaft ganz unvollkommen sey”. Impotenz in der Frühen Neuzeit. Diskurse und Praktiken in Deutschland. In: Österreichische Zeitschrift für Geschichtswissenschaft 22, N. 2 (2011), pp. 50-74.

Ruland, Martin: Curationum empiricarum et historicarum, in certis locis et notis personis optime expertarum et rite probatarum centuria prima. Basel 1578.

Russell, Andrew W. (ed.): The town and state physician in Europe from the Middle Ages to the Enlightenment. Wolfenbüttel 1981.

Sacchini, Franciscus: De ratione libros cum profectu legendi. Ingolstadt 1614.

Salatowsky, Sascha and Michael Stolberg (eds): Eine göttliche Kunst. Medizin und Krankheit in der Frühen Neuzeit (exhibition catalogue, Forschungsbibiothek Gotha). Gotha 2019.

Sander, Sabine: Handwerkschirurgen. Sozialgeschichte einer verdrängten Berufsgruppe. Göttingen 1989.

Santa Maria, Angiolgabriello di: Biblioteca, e storia di quei scrittori cosi della città come del territorio di Vicenza che pervennero fin' ad ora a notizia. Vol. 3 and Vol. 4. Vicenza 1772.

Santing, Catrien: Geneeskunde en humanisme. Een intellectuele biografie van Theodericus Ulsenius (c. 1460-1508). Rotterdam 1992.

Santoro, Marco: Uso e abuso delle dediche. A proposito del "Della dedicatione de' libri" di Giovanni Fratta. Rome 2006.

Sassonia, Ercole: Pantheum medicinae selectum: sive medicinae practicae templum, omnibus omnium fere morborum insultibus commune, libris undecim distinctum. Frankfurt 1603.

Savoia, Paolo: The book of the sick of Santa Maria della Morte in Bologna and the medical organization of a hospital in the sixteenth-century. In: Nuncius 31 (2016), pp. 163-235

Savonarola, Michele: Practica. Venice 1502.

Sawyer, Ronald C.: Friends or foes? Doctors and their patients in early modern England. In: Yosio Kawakita, Shizu Sakai and Yasuo Otsuka (eds.): History of the doctor-patient relationship. Tokyo/Brentwood 1995, pp. 31-53.

Sawyer, Ronald C.: Patients, healers, and disease in the Southeast Midlands, 1587-1634. Unpublished PhD-thesis, University of Wisconsin 1986.

Schäfer, Daniel: Regimina infantium. Die Sorge um die Gesundheit der Kinder in der Renaissance. In: Bergdolt, Hamm and Tönnesmann, Kind (2008), pp. 71-100.

Schaffrath, Ulrich: Läuse, Muscheln und Tabak. Das Herbar Ratzenberger. In: Philippia 15 (2012), pp. 191-214.

Schattner, Angela: Zwischen Familie, Heilern und Fürsorge. Das Bewältigungsverhalten von Epileptikern in deutschsprachigen Gebieten des 16.-18. Jahrhunderts. Stuttgart 2012. 
Schatzki, Theodore R., Karin Knorr Cetina and Eike von Savigny (eds): The practice turn in contemporary theory. London/New York 2001.

Schenck, Johann von Grafenberg: Observationum medicarum, rararum, novarum, admirabilium, et monstrosarum libri. 2 vols. Freiburg 1597/1599.

Schiebinger, Londa: Skeletons in the closet. The first illustrations of the female skeleton in eighteenth-century anatomy. In: Representations 14 (1986), pp. 42-82.

Schieß, Traugott: Briefe aus der Fremde von einem Zürcher Studenten der Medizin (Dr. Georg Keller) 1550-1558 (= Neujahrblatt Nr. 262). Zürich 1906.

Schild, Wolfgang: Das Blut des Hingerichteten. In: Christina von Braun and Christoph Wulf (eds): Mythen des Bluts. Frankfurt 2007, pp. 126-154.

Schilling, Ruth, Sabine Schlegelmilch and Susan Splinter: Stadtarzt oder Arzt in der Stadt? Drei Ärzte der Frühen Neuzeit und ihr Verständnis des städtischen Amtes. In: Medizinhistorisches Journal 46 (2011), pp. 99-133.

Schirrmeister, Albert: Triumph des Dichters. Gekrönte Intellektuelle im 16. Jahrhundert. Cologne/Weimar/Vienna 2003.

Schlegelmilch, Sabine: Ärztliche Praxis und sozialer Raum im 17. Jahrhundert. Johannes Magirus (1615-1697). Cologne 2018.

Schlegelmilch, Sabine: Das Selbstbewußtsein der Chirurgen. Tobias Geigers Traktat Discursus Medicus et Politicus (1656). In: Mariacarla Gadebusch Bondio, Christian Kaiser and Manuel Förg (eds): Menschennatur in Zeiten des Umbruchs. Das Ideal des "politischen" Arztes in der Frühen Neuzeit. Oldenburg 2020, pp. 141-176.

Schlegelmilch, Sabine: Promoting a good physician a town physician. Letters of application to German town authorities (1500-1700). In: Andrew Mendelsohn, Annemarie Kinzelbach and Ruth Schilling (eds): Civic medicine. Physician, polity, and pen in early modern Europe. Abingdon 2019, pp. 88-109.

Schlegelmilch, Sabine: "What a magnificent work a good physician is". The medical practice of Johannes Magirus (1615-1697). In: Dinges et alii, Medical practice (2016), pp. 151-168.

Schlegelmilch, Ulrich: Surgical disputations in Basel at around 1600. In: Meelis Friedenthal, Hanspeter Marti and Robert Seidel (eds): Early modern disputations and dissertations in an interdisciplinary and European context. Leiden 2021, pp. 255-287.

Schmid, Alois: "Poeta et orator a Caesare laureatus". Die Dichterkrönungen Kaiser Maximilians I. In: Historisches Jahrbuch 109 (1989), pp. 56-108.

Schmidt, Erich Ludwig: Deutsche Volkskunde im Zeitalter des Humanismus und der Reformation. Berlin 1904.

Schmidt, Paul Gerhard: Mediziner oder Poet? Soziale Lage und Lebenspläne hessischer Humanisten. In: August Buck and Tibor Klaniczay (eds): Sozialgeschichtliche Fragestellungen in der Renaissanceforschung. Wiesbaden 1992, pp.107-117.

Schmidt-Biggemann, Wilhelm: Topica universalis. Eine Modellgeschichte humanistischer und barocker Wissenschaft. Hamburg 1983.

Schmitt, Charles B.: Aristotle among the physicians. In: Wear, French and Lonie, Medical Renaissance (1985), pp. 1-15 and notes pp. 271-279.

Schmitt, Charles B.: Aristotle and the Renaissance. Cambridge 1983.

Schnell, Bernhard: Arzt und Literat. Zum Anteil der Ärzte am spätmittelalterlichen Literaturbetrieb. In: Sudhoffs Archiv 75 (1991), pp. 44-57.

Schober, Karl and Emil Neder: Sechshunderjahrfeier der Stadt Böhmisch Leipa, 1337-1937.

Böhmisch-Leipa 1929. 
Scholz, Lorenz: Catalogus arborum, fruticum et plantarum, tam indigenarum quam exoticarum, horti medici Laurentii Scholzii medici Vratisl. Breslau 1594.

Scholz, Lorenz (ed.): Consiliorum et epistolarum medicinalium [. . . ] liber secundus. Frankfurt 1592.

Scholz, Lorenz (Hrg.): Consiliorum et epistolarum medicinalium Ioannis Cratonis à Kraftheim liber septimus. Hannover 1611.

Scholz, Lorenz (ed.): Consiliorum medicinalium conscriptorum a praestantiss[imis] atque exercitatiss[imis] nostrorum temporum medicis liber singularis. Frankfurt 1598.

Scholz, Lorenz (ed.): Epistolarum philosophicarum, medicinalium ac chymicarum a summis nostrae aetatis philosophis ac medicis exaratum volumen. Frankfurt 1598.

Schrauf, Karl and Wenzel Hartl: Fünf Wiener Ärzte und Naturforscher aus dem XVI. Jahrhundert. Johann Aicholz, Diomedes Cornarius, Mathias Cornax, Wilhelm Coturnossius, Andreas Dadius. Vienna 1894.

Schrick, Michael: Von den ausgebrannten Wassern. Augsburg 1481.

Schuster, Daniel: “Die Festung des Lebens zu stürmen". Körper- und Krankheitsmetaphern in der medizinischen Ratgeberliteratur des 16. und 17. Jahrhunderts. Diss. med. Würzburg 2021.

Schütte, Jana Madlen: Medizin im Konflikt: Fakultäten, Märkte und Experten in deutschen Universitätsstädten des 14. bis 16. Jahrhunderts. Leiden 2017.

Schwarz, Christiane: Studien zur Stammbuchpraxis der Frühen Neuzeit. Gestaltung und Nutzung des Album amicorum am Beispiel eines Hofbeamten und Dichters, eines Politikers und eines Goldschmieds (etwa 1550-1650). Frankfurt 2002 (= Diss. phil. Munich 1999).

Scipio, Rosario (ed.): Girolamo Fabrici l'Acquapendente. Viterbo 1978.

Seidl, Katharina: “. . . how to assuage all outer and inner malady . . .". Medicine at the court of Archduke Ferdinand II. In: Sabine Haag and Veronika Sandbichler (eds): Ferdinand II. 450 years sovereign ruler of Tyrol. Exhibition catalogue. Innsbruck/Vienna 2017, pp. 67-71.

Senfelder, Leopold: Georg Handsch von Limus. Lebensbild eines Arztes aus dem XVI. Jahrhundert. In: Wiener klinische Rundschau (1901), pp. 495-499, pp. 514-516 and pp. 533-535.

Sennert, Daniel: Opera omnia. Lyon 1656.

Sennert, Daniel: Institutionum medicinae libri V. Wittenberg 1620.

Sevilla, Isidor of: Praeclarissimum opus [. . .] quod ethimologiarum intitulat[ur]. Paris 1509.

Sharpe, Kevin: The politics of reading in early modern England. New Haven 2000.

Sherman, W. H.: Used books. Marking readers in Renaissance England. Philadelphia 2008.

Sherrington, Charles: The endeavour of Jean Fernel. Cambridge 1946.

Simons, Madelon: “Een Theatrum van Representatie?” Aartshertog Ferdinand van Oostenrijk stadhouder in Praag tussen 1547 en 1567. Diss. phil. Amsterdam 2009.

Simons, Ronald C. and Charles C. Hughes (eds): The culture-bound syndromes. Folk illnesses of psychiatric and anthopological interest. Dordrecht 1985.

Siraisi, Nancy G.: Anatomizing the past. Physicians and history in Renaissance culture. In: Renaissance quarterly 53 (2000), pp. 1-30.

Siraisi, Nancy G.: Avicenna in Renaissance Italy. The "Canon" and medical teaching in Italian universities after 1500. Princeton 1987.

Siraisi, Nancy G.: Baudouin Ronsse as writer of medical letters. In: Ann Blair and Anja-Silvia Going (eds): For the sake of learning. Essays in honor of Anthony Grafton. Leiden/Boston 2016, pp. 123-139. 
Siraisi, Nancy G.: Communities of learned experience. Epistolary medicine in the Renaissance. Baltimore 2013.

Siraisi, Nancy G.: Die medizinische Fakultät. In: Walter Rüegg (ed.): Geschichte der Universität in Europa. Vol. I: Von der Reformation zur Französischen Revolution (1500-1800). Munich 1996, pp. 321-342.

Siraisi, Nancy G.: Disease and symptom as problematic concepts in Renaissance medicine. In: Eckhard Kessler and Ian Maclean (eds): Res et verba in der Renaissance. Wiesbaden 2002, pp. 217-240.

Siraisi, Nancy G.: History, medicine and the traditions of Renaissance learning. Ann Arbor 2007.

Siraisi, Nancy G.: L'individuale nella medicina tra medioevo e umanesimo: i 'casi clinici'. In: Roberto Cardini and Mariangela Regoliosi (eds): Umanesimo e medicina. Il problema dell'individuale. Rome 1996, pp. 33-62.

Siraisi, Nancy G.: Medicina practica. Girolamo Mercuriale as teacher and textbook author. In: Emidio Campi, Simone De Angelis, Anja-Silvia Goeing and Anthony T. Grafton (eds): Scholarly knowledge. Textbooks in early modern Europe. Geneva 2008, pp. 287-305.

Siraisi, Nancy G.: Medicine and the Italian universities 1250-1600. Leiden 2001.

Siraisi, Nancy G.: Medicine, 1450-1620, and the history of science. In: Isis 103 (2012), pp. 491-514.

Siraisi, Nancy G.: Medieval \& early Renaisance medicine. Chicago/London 1990.

Siraisi, Nancy G.: Oratory and rhetoric in Renaissance medicine. In: Journal of the history of ideas 65 (2004), pp. 191-211.

Siraisi, Nancy G.: Segni evidenti, teoria e testimonianza nelle narrazioni di autopsie del Rinascimento. In: Quaderni storici 36 (2001), pp. 719-744.

Siraisi, Nancy G.: Taddeo Alderotti and his pupils. Two generations of Italian medical learning. Princeton 1981.

Siraisi, Nancy G.: The clock and the mirror. Girolamo Cardano and Renaissance medicine. Princeton 1997.

Siraisi, Nancy G.: The faculty of medicine. In: Ridder-Symoens, History (1992), pp. 360-387.

Slater, John, and Maria Luz López Terrada: Scenes of mediation: Staging medicine in the Spanish interludes. In: Social history of medicine 24 (2011), pp. 226-243.

Smith, Pamela: The body of the artisan. Art and experience in the scientific revolution. Chicago/London 2004.

Smolka, Josef and Marta Vaculínová: Renesanční lékař Georg Handsch (1529-1578). In: DVT - Dějiny věd a techniky 43 (2010), pp. 1-26.

Sole, Brunoroa and Jacob Schultes: Loci communes juris caesarei, pontificii et saxonici. Opus legentibus, consulentibus, judicibus, advocatis utile, facile, necessarium. Leipzig 1607.

Solenander, Rainer: Consiliorum medicinalium Reineri Soleandri [. . . ] sectiones quinque [. . .] cum consiliis celeberrimi medici loannis Montani. $2^{\text {nd }}$ edn. Hannover 1609.

Spach, Israel (ed.): Gynaeciorum sive de mulierum tum communibus, tum gravidarum, parientium, et puerperarum affectibus et morbis libri. Strasbourg 1597.

Spach, Israel: Nomenclator scriptorum medicorum. Hoc est: Elenchus eorum, qui artem medicam suis scriptis illustrarunt, secundum locos communes ipsius medicinae. Strasbourg 1591.

Span, Lorenz: Epicedion nobili ac excellentissimo domino D. Andreæ de Gallis tridentino. Prague 1560.

Spitzer, Gabriele: Leonhard Thurneysser zum Thurn und die von ihm gegründete Berliner Druckerei (1574-1591). 3 vols. Diss. phil. Berlin 1987. 
Spitzner, Hermann Rudolf: Die Salernitanische Gynäkologie und Geburtshilfe unter dem Namen der "Trotula". Diss. Leipzig 1921.

Staden, Heinrich von: Incurability and hopelessness. The Hippocratic corpus. In: Paul Potter (ed.): La maladie et les maladies dans la collection hippocratique. Actes du Vle Colloque International Hippocratique. Québec 1990, pp. 75-112.

Starn, Randolph: A postmodern Renaissance? In: Renaissance quarterly 60 (2007), pp. 1-24.

Statuta Dominorum Artistarum Achademiae [sic] Patavina [Padua [?] c. 1600]

Stainpeiss, Martin: Liber de modo studendi seu legendi in medicina. Vienna 1520.

Starck, Andreas: Krancken Spiegel. Mülhausen 1598.

Steiger, Anselm Johann: Melancholie, Diätetik und Trost. Konzepte der Melancholie-Therapie im 16. und 17. Jahrhundert. Heidelberg 1996.

Stein, Claudia: Negotiating the French pox in early modern Germany. Farnham 2009.

Stengel, Johann: Theses de venae sectione. Würzburg 1602.

Sterzi, Giuseppe: Giulio Casseri anatomico e chirurgo (1552c.-1616). Venice 1909.

Stocker, Johannes: Empirica: sive medicamenta varia, experientia diuturna comprobata et stabilita, contra plerosque omnes corporis humani morbos tam internos quam externos. Ed. by Tobias Dornkrell ab Eberhertz. Frankfurt 1601.

Stöhsel, Robert: Die Fieberlehre an den Universitäten Montpellier und Pavia im 14. und 15. Jahrhundert. Mitteilung eines handschriftlichen "Sermo utilis de febribus" von Antonius Guaynerius. Diss. med. Würzburg 1923.

Stolberg, Michael: “Abhorreas pinguedinem": Fat and obesity in early modern medicine (c. 1500-1750) In: Studies in the history and philosophy of biology and biomedical sciences 43 (2012), pp. 370-378.

Stolberg, Michael, Accounting, religion, and the economics of medical care in $16^{\text {th }}$-century Germany. Hiob Finzel's Rationarium praxeos medicae, 1565-1589. In: Axel Hüntelmann and Oliver Falk (eds): Accounting for health. Calculation, paperwork, and medicine, 1500-2000. Manchester 2021, pp. 35-55.

Stolberg, Michael: Active euthanasia in early modern society. Learned debates and popular practices. In: Social history of medicine 20 (2007), pp. 205-221.

Stolberg, Michael: A sixteenth-century physician and his patients: The practice journal of Hiob Finzel, 1565-1589. In: Social history of medicine 32 (2019), pp. 221-240.

Stolberg, Michael: A woman down to her bones. The anatomy of sexual difference in the sixteenth and early seventeenth centuries. In: Isis 94 (2003), pp. 274-299.

Stolberg, Michael: A woman's hell? Medical perceptions of menopause in preindustrial Europe. In: Bulletin of the history of medicine 73 (1999), pp. 408-428.

Stolberg, Michael: Bedside teaching and the acquisition of practical skills in mid-sixteenthcentury Padua. In: Journal of the history of medicine and allied sciences 69 (2014), pp. 633-661.

Stolberg, Michael: “Cura palliativa”. Begriff und Diskussion der palliativen Krankheitsbehandlung in der vormodernen Medizin (ca. 1500-1850). In: Medizinhistorisches Journal 42 (2007), pp. 7-29.

Stolberg, Michael: Der gesunde Leib. Zur Geschichtlichkeit frühneuzeitlicher Körpererfahrung. In: Paul Münch (ed.): "Erfahrung" als Kategorie der Frühneuzeitgeschichte (= Historische Zeitschrift, Beiheft 31 (2001)), pp. 37-57.

Stolberg, Michael: Die Geschichte der Palliativmedizin. Medizinische Sterbebegleitung von 1500 bis heute. Frankfurt 2011. 
Stolberg, Michael: Die Homöopathie auf dem Prüfstand. Der erste Doppelblindversuch der Medizingeschichte im Jahr 1835. In: Münchener Medizinische Wochenschrift 138 (1996), pp. 364-366.

Stolberg, Michael: Emotions and the body in early modern medicine. In: Emotion review 11 (2019), pp. 113-122.

Stolberg, Michael: Empiricism in sixteenth-century medical practice. The notebooks of Georg Handsch. In: Early science and medicine 18 (2013), pp. 487-516.

Stolberg, Michael: Enthüllungen. Die uroskopische Schwangerschaftsdiagnose und ihre Darstellung in der frühneuzeitlichen Kunst. In: Daniel Hornuff and Heiner Fangerau (eds): Visualisierung des Ungeborenen. Paderborn 2020, pp. 51-68.

Stolberg, Michael: Erfahrungen und Deutungen der weiblichen Monatsblutung in der Frühen Neuzeit. In: Barbara Mahlmann-Bauer (ed.): Scientiae et artes. Die Vermittlung alten und neuen Wissens in Literatur, Kunst und Musik. Wolfenbüttel 2004, pp. 913-931.

Stolberg, Michael: Examining the body (c. 1500-1750) In: Sarah Toulalan and Kate Fisher (eds): The Routledge history of sex and the body, 1500 to the present. Oxford 2013, pp. 91-105.

Stolberg, Michael: Experiencing illness and the sick body in early modern Europe. London 2011.

Stolberg, Michael: Formen und Funktionen ärztlicher Fallberichte in der Frühen Neuzeit (1500-1800). In: Johannes Süßmann, Susanne Scholz and Gisela Engel (eds): Fallstudien: Theorie - Geschichte - Methode. Berlin 2007, pp. 81-95.

Stolberg, Michael: Heilkunde zwischen Staat und Bevölkerung. Angebot und Annahme medizinischer Versorgung in Oberfranken im frühen 19. Jahrhundert. Diss. med. TU Munich 1986.

Stolberg, Michael: Metaphors and images of cancer in early modern Europe. In: Bulletin of the history of medicine 88 (2014), pp. 48-74.

Stolberg, Michael: Keeping the body open. Impurity, excretions, and healthy living in the early modern period. In: James Kennaway and Rina Knoeff (eds): Lifestyle and medicine in the Enlightenment. The six non-naturals in the long eighteenth century. New York/London 2020, pp. 205-222.

Stolberg, Michael: Kommunikative Praktiken. Ärztliche Wissensvermittlung am Krankenbett im 16. Jahrhundert. In: Arndt Brendecke (ed.): Praktiken der Frühen Neuzeit. Akteure Handlungen - Artefakte. Cologne/Weimar/Vienna 2015, pp. 111-121.

Stolberg, Michael: Konservierte Pflanzen für die Wissenschaft. In: Salatowsky/Stolberg, Göttliche Kunst (2019), pp. 122-124.

Stolberg, Michael: Krankheitsgeschehen und leibärztliche Praxis am Hof von Erzherzog Ferdinand II. Die Aufzeichnungen des Georg Handsch (1529-1578). In: Elena Taddei and Marina Hilber (eds): In fürstlicher Nähe. Ärzte bei Hof (1450-1800). Innsbruck 2021, pp. 91-110.

Stolberg, Michael: Learning anatomy in late sixteenth-century Padua. In: History of science 56 (2018), pp. 381-402.

Stolberg, Michael: Learning from the common folks. Academic physicians and medical lay culture in the sixteenth century. In: Social history of medicine 27 (2014), pp. 649-667.

Stolberg, Michael: Lykanthropie. In: Manfred Landfester (ed.): Der Neue Pauly. Vol. 15/1: Wissenschafts- und Rezeptionsgeschichte La-Ot. Stuttgart/Weimar 2001, pp. 243-246.

Stolberg, Michael: Medical note-taking in the sixteenth and seventeenth centuries. In: Alberto Cevolini (ed.): Forgetting machines: Knowledge management evolution in early modern Europe. Leiden/Boston 2016, pp. 243-264. 
Stolberg, Michael: Medizinische Loci communes. Formen und Funktionen einer ärztlichen Aufzeichnungspraxis im 16. und 17. Jahrhundert. In: NTM - Zeitschrift für Geschichte der Wissenschaften, Technik und Medizin 21 (2013), pp. 37-60.

Stolberg, Michael: “Mein askulapisches Orakel!”: Patientenbriefe als Quelle einer Kulturgeschichte der Krankheitserfahrung im 18. Jahrhundert. In: Österreichische Zeitschrift für Geschichtswissenschaft 7 (1996), pp. 385-404.

Stolberg, Michael: Menstruation and sexual difference in early modern medicine. In: Andrew Shail and Gillian Howie (eds): Menstruation. A cultural history. Basingstoke 2005, pp. 90-101.

Stolberg, Michael: Metaphors and images of cancer in early modern Europe. In: Bulletin of the history of medicine 88 (2014), pp. 48-74.

Stolberg, Michael: Möglichkeiten und Grenzen einer retrospektiven Diagnose. In: Waltraud Pulz (ed.): Zwischen Himmel und Erde. Körperliche Zeichen der Heiligkeit. Stuttgart 2012, pp. 209-227.

Stolberg, Michael: Negotiating the meanings of illness. Medical popularization and the patient in the $18^{\text {th }}$ century. In: Wilhelm de Blécourt and Cornelie Usborne (eds): Cultural approaches to the history of medicine. Mediating medicine in early modern and modern Europe. Basingstoke/New York 2004, pp.89-107.

Stolberg, Michael: Post-mortems, anatomical dissections and humoural pathology in the sixteenth and early seventeenth centuries. In: Silvia De Renzi, Marco Bresadola and Maria Conforti (eds): Pathology in practice. Diseases and dissections in early modern Europe. New York/London 2017, pp. 79-95.

Stolberg, Michael: Probleme und Perspektiven einer Geschichte der Volksmedizin. In: Thomas Schnalke and Claudia Wiesemann (eds): Die Grenzen des Anderen. Medizingeschichte aus postmoderner Perspektive. Vienna 1998, pp. 49-73.

Stolberg, Michael: Studying medicine in $16^{\text {th }}$-century Padua and Montpellier. A comparative analysis from the perspectives of medical students. In: Delia Gavrus and Susan Lamb (eds): History of medical education. Montreal 2022 [in preparation].

Stolberg, Michael: Sweat. Learned concepts and popular perceptions, 1500-1800. In: Manfred Horstmannshoff, Helen King and Claus Zittel (eds): Blood, sweat and tears. The changing concepts of physiology from antiquity to early modern Europe. Leiden/Boston 2012, pp. 503-522.

Stolberg, Michael: Teaching anatomy in post-Vesalian Padua. An analysis of student notes. In: Journal of medieval and early modern studies 48 (2018), pp. 61-78.

Stolberg, Michael: The decline of uroscopy in early modern learned medicine, 1500-1650. In: Early science and medicine 12 (2007), pp. 313-336.

Stolberg, Michael: The doctor-patient relationship in the Renaissance. In: European journal for the history of medicine and health 1 (2021), pp. 1-29, open access: https://doi.org/ 10.1163/26667711-bja10001

Stolberg, Michael: The many uses of writing. A humanist physician in sixteenth-century Prague. In: Andrew Mendelsohn, Annemarie Kinzelbach and Ruth Schilling (eds): Civic medicine. Physician, polity, and pen in early modern Europe. London 2019, pp. 67-87.

Stolberg, Michael: The monthly malady: A history of premenstrual suffering. In: Medical history 44 (2000), pp. 301-322.

Stolberg, Michael: Tödliche Menschenversuche im 16. Jahrhundert. In: Deutsches Ärzteblatt, Ausgabe A 111 (2014), pp. 2060-2062.

Stolberg, Michael: Uroscopy in early modern Europe. Farnham/ Burlington, VT, 2015. 
Stolberg, Michael: "You have no good blood in your body". Oral communication in sixteenth-century physicians’ medical practice. In: Medical history 59 (2015), pp. 63-82.

Stolberg, Michael: “Zorn, Wein und Weiber verderben unsere Leiber." Krankheit und Affekt in der frühneuzeitlichen Medizin. In: Johann Anselm Steiger and Ralf Georg Bogner (eds): Passion, Affekt und Leidenschaft in der Frühen Neuzeit. Wiesbaden 2005, pp. 1033-1059.

Stolberg, Michael: Zwischen Identitätsbildung und Selbstinszenierung. Ärztliches SelfFashioning in der Frühen Neuzeit. In: Dagmar Freist (ed.): Diskurse - Körper - Artefakte. Historische Praxeologie in der Frühneuzeitforschung. Bielefeld 2015, pp. 33-55.

Stolberg, Michael and Tilmann Walter: Martin Luthers viele Krankheiten. Ein unbekanntes Konsil von Matthäus Ratzenberger und die Problematik der retrospektiven Diagnose. In: Archiv für Reformationsgeschichte 109 (2018), pp. 126-151.

Stolz, Michael: Artes-liberales-Zyklen: Formationen des Wissens im Mittelalter. Vol. 1. Tübingen/Basel 2004.

Storchová, Lucie: Bohemian school humanism and its editorial practices (ca. 1550-1610). Turnhout 2014.

Storchová, Lucie: Collinus, Matthaeus. In: eadem (ed.): Companion to Central and Eastern European humanism. Vol. 2: Czech lands, part 1: A-L. Berlin 2020, pp. 298-316.

Storchová, Lucie: Handsch, Georg. In: eadem (ed.): Companion to Central and Eastern European humanism. Vol. 2: Czech lands, part 1: A-L. Berlin 2020, pp. 512-522.

Storchová, Lucie: Humanist occasional poetry and strategies for acquiring patronage. The case of Georg Handsch. In: Sylva Dobalová and Jaroslava Hausenblasová: Archduke Ferdinand Il of Austria: A second-born son in Renaissance Europe [in preparation].

Storchová, Lucie: Paupertate styloque connecti. Utváření humanistické učenecké komunity $v$ českých zemích. Prague 2011.

Storchová, Lucie: "The tempting girl, I know so well": Representations of gout and the self-fashioning of Bohemian humanist scholars. In: Early science and medicine 21 (2016), pp. 511-530.

Strobelberger, Johann Stefan: Laureationum medicarum apud exteros promeritarum adversum obtrectatores breves vindiciae, in honorem Scholae medicae Monspeliensis propositae. Nürnberg 1628.

Stroh, Walter: Aerztliche Bewerbungen, Berufungen, Bestallungen des 15. und des 16. Jahrhunderts, aus Esslingen, sowie Verwandtes zum ärztlichen Standeswesen jener Zeit. Diss. med. Leipzig 1920.

Stromer, Heinrich: Algorithmus linealis numerationem, additionem, subtractionem, duplationem, mediationem, multiplicationem, divisionem et progressionem una cum regula de tri perstringens. Vienna 1520.

Stübler, Eberhard: Geschichte der medizinischen Fakultät der Universität Heidelberg 1386-1925. Heidelberg 1926.

Stupanus, Johann Niklaus: De praefocatione matricis. Exhibet Rudolph Heinrich Groshaus. Basel 1612.

Sudhoff, Karl: Erstlinge der pädiatrischen Literatur. Drei Wiegendrucke über Heilung und Pflege des Kindes. Munich 1925.

Sudhoff, Karl: latromathematiker vornehmlich im 15. und 16. Jahrhundert. Breslau 1902.

Sudhoff, Karl: Kurpfuscher, Ärzte und Stadtbehörden am Ende des 15. Jahrhunderts. Handschriften- und Aktenstudie. In: Archiv für Geschichte der Medizin 8 (1915), pp. 98-124. 
Sudhoff, Karl: Versuch einer Kritik der Echtheit der Paracelsischen Schriften. 2 vols. Berlin $1898 / 99$.

Sudhoff, Karl: Zur Geschichte der Lehre von den kritischen Tagen im Krankheitsverlaufe. In: Sudhoffs Archiv 21 (1929), pp. 1-22.

Sullivan, Erin: Beyond melancholy. Sadness and selfhood in Renaissance England. Oxford 2016.

Svobodný, Petr: The medical faculty. In: Ivana Čornejová and Michal Svatoš (eds): A history of Prague University, 1348-1802. Prague 2001, pp. 171-185.

Sylvius, Jacobus: Ordo et ordinis ratio in legendis Hippocratis et Galeni libris. Paris 1548.

Talbot, Charles: Medical education in the Middle Ages. In: O’Malley, History (1970), pp. 73-87.

Tanfani, Gustavo: "I consilia medica" di Vittore Trincavella. In: Rivista di storia delle scienze mediche e naturali 43 (1952), pp. 248-254.

Tanner, Jakob: Historische Anthropologie zur Einführung. Hamburg 2004.

Tannstetter, Georg: Artificium de applicatione astrologiae ad medicinam, deque conviventia earundem. Ed. and comm. by Rosemarie Eichinger. Vienna/Münster 2006 (orig.:

Strasbourg 1531).

Telle, Joachim: Arzneikunst und der "gemeine Mann". Zum deutsch-lateinischen Sprachenstreit in der frühneuzeitlichen Medizin. In: Herzog August Bibliothek Wolfenbüttel, (ed.): Pharmazie und der gemeine Mann. Hausarznei und Apotheke in deutschen Schriften der frühen Neuzeit. Wolfenbüttel 1982, pp. 43-48.

Telle, Joachim: Bartholomäus Carrichter. Zu Leben und Werk eines deutschen Fachschriftstellers des 16. Jahrhunderts. Mit einem Werkverzeichnis von Julian Paulus. In: Daphnis, Zeitschrift für mittlere deutsche Literatur 26 (1997), pp. 715-751.

Temkin, Owsei: An historical analysis of the concept of infection. In: idem: Studies in intellectual history. Baltimore 1968, pp. 123-147.

Temkin, Owsei: Fernel, Joubert, and Erastus on the specificity of cathartic drugs. In: Allen G. Debus (ed.): Science, medicine and society in the Renaissance. Vol. 1. New York 1972, pp. 61-68.

Temkin, Owsei: Galenism: Rise and decline of a medical philosophy. Ithaca, N.Y. 1973.

Temkin, Owsei: Studien zum “Sinn”-Begriff in der Medizin. In: Kyklos 2 (1929), pp. 21-105.

Temkin, Owsei: The falling sickness. A history of epilepsy from the Greeks to the beginnings of modern neurology. $2^{\text {nd }}$ edn. Baltimore/London 1971.

Theodosius, Ioannes Baptista: Medicinales epistolae LXVIIII. Basel 1553.

Thoner, Augustinus: Observationum medicinalium haud trivialium libri quatuor. Ulm 1649.

Thurn, Nikolaus: Deutsche neulateinische Städtelobgedichte. Ein Vergleich ausgewählter Beispiele des 16. Jahrhunderts. In: Neulateinisches Jahrbuch 4 (2002), pp. 253-269.

Thurneisser zum Thurn, Leonhard: Pison. Das erst Theil. Von kalten, warmen minerischen und metallischen Wassern, sampt der Vergleichunge der Plantarum und Erdgewechsen 10. Bücher. Frankfurt an der Oder 1572.

Timaeus von Güldenklee, Balthasar: Casus medicinales praxi triginta sex annorum observati. Leipzig 1667.

Timaeus von Güldenklee, Balthasar: Casus et observationes practicae triginta sex annorum. Leipzig 1691.

Timaeus von Güldenklee, Balthasar: Responsa medica et diaeteticon opus posthumum. Leipzig 1668.

Tiraboschi, Girolamo: Biblioteca modenese. Vol. II. Modena 1782. 
Touwaide, Alain: Galien et la toxicologie. In: Wolfgang Haase (ed.): Aufstieg und Niedergang der römischen Welt. Part II: Principat. Vol. 37,2. Berlin/New York 1994, pp. 1887-1986.

Tovazzi, Giangrisostomo: Familiarium tridentinum. Trento 2006 (http://www.db.ofmtn.pcn. net/ofmtn/files/biblioteca/TOVAZZI\%20FAMILIARIUM\%20TRIDENTINUM.pdf).

Toxites, Michael: Spongia stibii adversus Lucae Stenglini medicinae doctoris et physici augustani aspergines. Strasbourg 1567.

Traister, Barbara Howard: The notorious astrological physician of London. Works and days of Simon Forman. Chicago/London 2001.

Trevor-Roper, Hugh: The court physician and Paracelsism. In: Nutton, Medicine (1990), pp. 79-94.

Triebs, Michaela: Die Medizinische Fakultät der Universität Helmstedt (1576-1810). Eine Studie zu ihrer Geschichte unter besonderer Berücksichtigung der Promotions- und Übungsdisputation. Wiesbaden 1995.

Trincavella, Vettore: Consilia medica post editionem venetam et lugdunensem, accessione CXXVIII consiliorum locupletata, et per locos communes digesta. Basel 1587.

Trincavella, Vettore: Consiliorum medicinalium libri III. Epistolarum medicinalium libri III. Venice 1586.

Trincavella, Vettore: De ratione curandi particulares humani corporis affectus praelectiones. Venice 1575.

Truc, Miroslav: Die gesellschaftliche Aufgabe der Prager Karls-Universität in der zweiten Hälfte des 16. und am Anfang des 17. Jahrhunderts. In: Hans-Bernd Harder (ed.): Später Humanismus in der Krone Böhmens 1570-1620. Dresden 1998, pp. 203-210.

Tulpius, Nicolaus: Observationum medicarum libri tres. Amsterdam 1641.

Uhlig, Paul: Auf der Suche nach Stadtärzten: Zwickauer Ratsprotokolle berichten. In: Sudhoffs Archiv für Geschichte der Medizin und der Naturwissenschaften 31 (1938), pp. 330-336.

Ullmann, Manfred: Die Medizin im Islam. Leiden/Cologne 1970.

Valleriola, François: Loci medicinae communes, tribus libris digesti. Lyon 1562.

Valleriola, François: Loci medicinae communes, tribus libris digesti. Venice 1563.

Valleriola, François: Loci medicinae communes, tribus libris digesti, quibus accessit appendix, universa complectens ea, quae ad totius operis integritatem deesse videbantur. Lyon 1589.

Valleriola, François: Observationum medicinalium libri sex. Lyon 1573.

Vanzan-Marchini, Nelli-Elena: I mali e i rimedi della serenissima. Venice 1995.

Varanda, Jean: De morbis mulierum libri III. Montpellier 1620.

Vekerdy, Lilla: Paracelsus's Great Wound Surgery. In: Elizabeth Lane Furdell (ed.): Textual healing. Essays on medieval and early modern medicine. Leiden/Boston 2005, pp. 77-99.

Vesal, Andreas: De humani corporis fabrica. Basel 1543.

Vieler, Ingrid: Die deutsche Arztpraxis im 19. Jahrhundert. Diss. med. Mainz 1958.

Vietor, Peter: Theses medicae de praefocatione uteri. Basel 1610.

Vigo, Giovanni da: The most excellent workes of chirurgerye. [London] 1543.

Vinař, Josef: Obrazy z minulosti českého lékářství. Prague 1959.

Vischer, Christoph: Die Stammbücher der Universitätsbibliothek Basel: ein beschreibendes

Verz[eichnis]. In: Festschrift Karl Schwarber. Basel 1949, pp.247-264.

Vittore, Benedetto: Medicatio empirica singulorum morborum. Paris 1551.

Vittori, Leonello: De aegritudinibus infantium tractatus admodum salutifer. Venice 1557.

Vives, Juan Luís: De conscribendis epistolis. Basel 1536. 
Vives, Juan Luís: The passions of the soul (1543). Ed. by Carlos G. Noreña. Lewiston/Queenston 1990.

Voigtlaender, Heinz: Löhne und Preise in vier Jahrtausenden. Speyer 1994.

Waardt, Hans de: Johann Wier. Hofarzt von Herzog Wilhelm und Vorkämpfer für Toleranz. In: Guido von Büren, Ralf-Peter Fuchs and Georg Mölich (eds): Herrschaft, Hof und Humanismus. Wilhelm V. von Jülich-Kleve-Berg und seine Zeit. Bielefeld 2018, pp. 573-590.

Wackerbauer, Anton: Dr. Reiner Solenander (Reinhard Gathmann) ein niederrheinischer Arzt, Leibarzt am Düsseldorfer Hofe (1524-1601). In: Düsseldorfer Jahrbuch. Beiträge zur Geschichte des Niederrheins 37 (1932/33), pp. 95-140.

Wagner, Wolfgang Eric: Doctores - Practicantes - Empirici. Die Durchsetzung der Medizinischen Fakultäten gegenüber anderen Heilergruppen in Paris und Wien im späten Mittellalter. In: Rainer C. Schwinges (ed.): Universität im öffentlichen Raum. Basel 2008, pp. 15-43.

Wallis, Patrick: Competition and cooperation in the early modern medical economy. In: Mark

S. R. Jenner and Patrick Wallis (eds): Medicine and the market in England and its colonies, c. 1450-c. 1850. London 2007, pp. 47-68.

Walter, Tilmann: Ärztliche Selbstdarstellung im Zeitalter der Fugger und Welser. Epistolarische Strategien und Repräsentationspraktiken bei Felix Platter (1536-1614). In: Angelika Westermann and Stefanie von Welser (eds): Personen und Milieu. Individualbewusstsein? Persönliches Profil und soziales Umfeld. Husum 2013, pp. 285-314.

Walter, Tilmann: Ärztehaushalte im 16. Jahrhundert. Einkünfte, Status und Praktiken der Repräsentation. In: Medizin, Geschichte und Gesellschaft 27 (2008), pp. 31-73.

Walter, Tilmann: New light on Antiparacelsianism (c. 1570-1610). The medical republic of letters and the idea of progress in science. In: Sixteenth century journal 43 (2012), pp. 701-725.

Wear, Andrew: Explorations in Renaissance writings on the practice of medicine. In: Wear, French and Lonie, Medical Renaissance (1985), pp. 118-145.

Wear, Andrew: Knowledge \& practice in English medicine, 1550-1680. Cambridge 2000.

Wear, Andrew: Medical practice in late seventeenth- and early eighteenth-century England: Continuity and union. In: idem, R. K. French and I. M. Lonie (eds): The medical revolution of the seventeenth century. Cambridge 1989, pp. 294-320.

Wear, Andrew: Medicine in early modern Europe 1500-1700. In: Conrad Lawrence, Michael Neve, Vivian Nutton, Roy Porter and Andrew Wear: The Western medical tradition, 800 BC to $A D$ 1800. Cambridge, 1995, pp. 215-361.

Wear, Andrew: Popularized ideas of health and illness in seventeenth-century France. In: Seventeenth century French studies 8 (1986), pp. 229-242.

Wear, Andrew: The spleen in Renaissance anatomy. In: Medical history 21 (1977), pp. 43-60. Wear, Andrea, Roger French and lain Lonie (eds): The medical Renaissance of the sixteenth century. Cambridge 1985.

Webster, Charles: Paracelsus: Medicine, magic and mission at the end of time. New Haven/ London 2008.

Wehrli, Gustav A.: Die Bader, Barbiere und Wundärzte im alten Zürich. Zürich 1927.

Wehrli, Gustav: Der Zürcher Stadtarzt Dr. Christoph Clauser und seine Stellung zur Reformation der Heilkunde im 16. Jahrhundert. Nebst Faksimileausgabe seiner Harnschrift und seiner Kalender. Zürich 1924. 
Weisser, Olivia: Ill composed. Sickness, gender, and belief in early modern England. New Haven/London 2015.

Wellner, Axel: Bergmedicus Christian August Mithoff (1615-1657). Ein Beitrag zur Medizingeschichte des Harzes. In: Allgemeiner Harz-Berg-Kalender für das Jahr 1984 (1984), pp. 36-38.

Welsch, Georg Hieronymus: Curationum exotericarum chiliades II. Ulm 1676. Welsch, Georg Hieronymus: Consiliorum medicinalium centuriae IV. UIm 1676. Weston, Robert: Medical consulting by letter in France, 1665-1789. Farnham 2013. Weyer, Johannes: De praestigiis daemonum libri V. Basel 1564.

Widmann, Martin and Christoph Mörgeli: Bader und Wundarzt. Medizinisches Handwerk in vergangenen Tagen. Zürich 1998.

Wiedemann, Theodor: Geschichte der Reformation und Gegenreformation im Lande unter der Enns. Vol. 3: Die reformatorische Bewegung im Bisthume Passau. Prague 1882.

Wiegand, Hermann: Volkskunde und Ethnographie bei Konrad Celtis. In: Franz Fuchs (ed.): Konrad Celtis in Nürnberg. Wiesbaden 2004, pp. 51-73.

Wightman, William P.D.: Quid sit methodus? "Method” in the sixteenth century medical teaching and "discovery". In: Journal of the history of medicine 19 (1964), pp. 360-376.

Williams, Katherine E.: Hysteria in seventeenth-century case records and unpublished manuscripts. In: History of psychiatry 1 (1990), pp. 383-401.

Wilson, Adrian: On the history of disease concepts. The case of pleurisy. In: History of science 38 (2000), pp. 271-319.

Wittern, Wittern: Die Unterlassung ärztlicher Hilfeleistung in der griechischen Medizin der klassischen Zeit. In: Münchener medizinische Wochenschrift 121 (1979), pp. 731-734.

Wittich, Johannes: Praeservator sanitatis. Ein nützlicher Bericht von den sechs unvormeidlichen [sic] Dingen, zur Gesundheit gantz ersprießlichen, wie man sich in denselben beydes zu Hause und auch über Land verhalten sol. Leipzig 1590.

Wittstock, Antje: Melancholia translata: Marsilio Ficinos Melancholie-Begriff im deutschsprachigen Raum des 16. Jahrhunderts. Göttingen 2011.

Wolf, Kaspar (ed.): Gynaeciorum, hoc est, de mulierum tum communibus, tum aliis, tum gravidarum, parientium, et puerperarum affectibus et morbis libri. Basel 1566.

Wolff, Eberhard: "Volksmedizin": Abschied auf Raten. Vom definitorischen zum heuristischen Begriffsverständnis. In: Zeitschrift für Volkskunde 94 (1998), pp. 233-257.

Wolff, Fritz: Kartographen - Autographen (exhibition catalogue, Hessisches Staatsarchiv in Marburg). Marburg 1990.

Wolff, Jacob: Die Lehre von der Krebskrankheit von den ältesten Zeiten bis zur Gegenwart. Vol. 1, $2^{\text {nd }}$ edn. Jena 1929.

Wolfangel, Doris: Dr. Melchior Ayrer (1520-1579). Diss. med. Würzburg 1957.

Wolkan, Rudolf: Geschichte der deutschen Litteratur in Boehmen bis zum Ausgange des XVI. Jahrhunderts. Prague 1894.

Wolkan, Rudolf: Geschichte der deutschen Literatur in Böhmen und in den Sudentenländern. Augsburg 1925.

Wolkan, Rudolf: Handsch, Georg. In: Allgemeine Deutsche Biographie. Vol. 49 (1904), pp. 749-751.

Wondrák, Eduard: Der Arzt und Dichter Laurentius Span (1530-1575). In: Medizinhistorisches Journal 18 (1983), pp. 238-249.

Worstbrock, Franz Josef (ed.): Der Brief im Zeitalter der Renaissance. Weinheim 1983. 
Wotschke, Theodor: Johann Theobald Blasius, ein Lissaer Rektor des 16. Jahrhunderts. In:

Deutsche Wissenschaftliche Zeitschrift für Polen 6 (1925), pp. 1-30.

Wustmann, Gustav: Der Wirt von Auerbachs Keller. Dr. Heinrich Stromer von Auerbach

(1482-1542). Mit sieben Briefen Stromers an Spalatin. Berlin 1902.

Yeo, Richard: Notebooks, English virtuosi, and early modern science. Chicago/London 2014.

Zacchia, Paolo: Quaestiones medico-legales. $3^{\text {rd }}$ edn. Amsterdam 1651.

Zahn, G.: Das Herbar des Dr. Caspar Ratzenberger (1598) in der Herzoglichen Bibliothek zu Gotha. In: Mitteilungen des Thüringischen Botanischen Vereins. N.F. 16 (1901), pp. 50-121.

Zaunick, Rudolph: Beiträge zur Geschichte der Leipziger chirurgisch-anatomischen Professur vor 1580. In: Archiv für Geschichte der Medizin 16 (1924/25), pp. 189-208.

Zedelmaier, Helmut: Navigieren im Text-Universum. Theodor Zwingers Theatrum vitae humanae. In: metaphorik.de 14 (2008), pp. 113-135.

Zerbi, Gabriele: Opus perutile de cautelis medicorum. [Venice, after 1494].

Zinn, Johann Conrad: Disputatio de vulneribus capitis. Basel 1595.

Zitter, Miriam: Die Leibärzte der württembergischen Grafen im 15. Jahrhundert (1397-1496). Zur Medizin an den Höfen von Eberhard dem Milden bis Eberhard im Bart. Leinfelden/ Echterdingen 2000.

Zwinger, Theodor: Theatrum vitae humanae. Basel 1586. 
